\title{
Engineering generalized Gibbs ensembles with trapped ions
}

\author{
Florentin Reiter $\odot,{ }^{1,2}$ Florian Lange $\odot,{ }^{3}$ Shreyans Jain $\odot,{ }^{2}$ Matt Grau $\odot,{ }^{2}$ Jonathan P. Home, ${ }^{2}$ and Zala Lenarčič $\odot 4,5$ \\ ${ }^{1}$ Harvard University, Department of Physics, Cambridge, Massachusetts 02138, USA \\ ${ }^{2}$ Institute for Quantum Electronics, ETH Zürich, Otto-Stern-Weg 1, 8093 Zürich, Switzerland \\ ${ }^{3}$ Institute for Theoretical Physics, University of Cologne, D-50937 Cologne, Germany \\ ${ }^{4}$ Department of Physics, University of California, Berkeley, California 94720, USA \\ ${ }^{5}$ Jožef Stefan Institute, 1000 Ljubljana, Slovenia
}

(Received 30 October 2020; accepted 23 April 2021; published 12 August 2021)

\begin{abstract}
The concept of generalized Gibbs ensembles (GGEs) has been introduced to describe steady states of integrable models. Recent advances show that GGEs can also be stabilized in nearly integrable quantum systems when driven by external fields and open. Here, we present a weakly dissipative dynamics that drives towards a steady-state GGE and is realistic to implement in systems of trapped ions. We outline the engineering of the desired dissipation by a combination of couplings which can be realized with ion-trap setups and discuss the experimental observables needed to detect a deviation from a thermal state. We present a mixed-species motional mode engineering technique in an array of microtraps and demonstrate the possibility to use sympathetic cooling to construct many-body dissipators. Our paper provides a blueprint for experimental observation of GGEs in open systems and opens an avenue for quantum simulation of driven-dissipative quantum many-body problems.
\end{abstract}

DOI: 10.1103/PhysRevResearch.3.033142

\section{INTRODUCTION}

Providing a compact description of complicated manybody systems is a challenging task. Studies of equilibration of interacting quantum many-body systems have revealed that one can borrow statistical descriptions, taking into account conservation laws of equilibrating systems, to achieve that $[1,2]$. If we suddenly excite an ergodic system, for which energy is the only conservation law, steady-state expectation values will be given by a Gibbs ensemble

$$
\rho_{\mathrm{th}}=\frac{e^{-\beta H_{0}}}{\operatorname{tr}\left[e^{-\beta H_{0}}\right]}
$$

with temperature $1 / \beta$ determined by the amount of energy that has been injected into the system with the excitation process [1]. Such a description by the single parametertemperature-is, therefore, an incredible simplification for an interacting model with a priori exponentially many degrees of freedom.

Similarly, generalized Gibbs ensembles (GGEs) were proposed to describe local observables in steady states of systems with additional local conservation laws $C_{i}$ (see Fig. 1). The existence of additional conservation laws highly restricts the dynamics and prevents the system from thermalizing. GGEs have a form related to that of a Gibbs ensemble [3],

$$
\rho_{\mathrm{GGE}}=\frac{e^{-\sum_{i} \lambda_{i} C_{i}}}{\operatorname{tr}\left[e^{-\sum_{i} \lambda_{i} C_{i}}\right]},
$$

Published by the American Physical Society under the terms of the Creative Commons Attribution 4.0 International license. Further distribution of this work must maintain attribution to the author(s) and the published article's title, journal citation, and DOI. but with additional Lagrange multipliers $\lambda_{i}$ associated with additional conserved quantities $C_{i}$ that all commute with the Hamiltonian, $\left[C_{i}, H_{0}\right]=0$. Exemplary models with macroscopically many local conservation laws, where
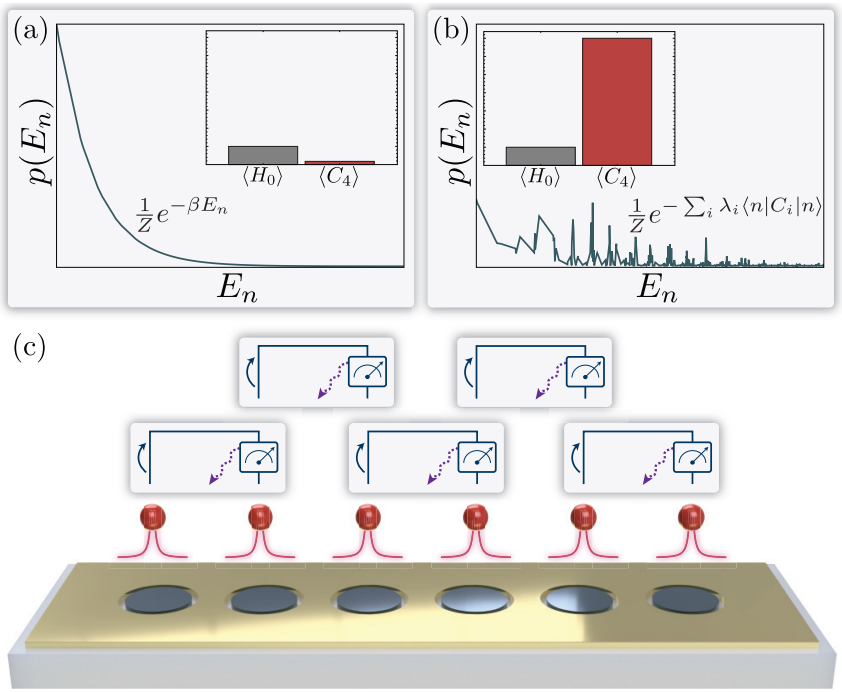

FIG. 1. Activating integrability. (a) Following an excitation of a nonintegrable or nearly integrable system, the steady state exhibits thermal behavior with small expectation values of operators other than the Hamiltonian. (b) A nearly integrable system with a weak dissipative drive will show highly nonthermal behavior with a distribution $p\left(E_{n}\right)$ approximately described by a GGE and large expectation values for conservation laws, e.g., $\left\langle C_{4}\right\rangle$. (c) $\mathrm{A}$ onedimensional array of trapped ions has interactions which decay as a power law, and is thus nearly integrable for large exponents. We reactivate integrable effects by engineering the dissipation, which stabilizes a GGE as the steady state. 
applicability of GGE has been widely studied theoretically, are Bethe-Ansatz-solvable and noninteracting integrable systems [4-25]. In this case, Lagrange parameters $\lambda_{i}$ are fixed by the knowledge of the initial state $|\psi(0)\rangle$, as well as $\left\langle C_{i}\right\rangle=$ const:

$$
\left\langle\psi(0)\left|C_{k}\right| \psi(0)\right\rangle \stackrel{!}{=} \operatorname{tr}\left[C_{k} \frac{e^{-\sum_{i} \lambda_{i} C_{i}}}{\operatorname{tr}\left[e^{-\sum_{i} \lambda_{i} C_{i}}\right]}\right], \quad \forall k .
$$

Since generically $\lambda_{i} \neq 0$, integrable systems remain nonthermal up to arbitrary times.

Applicability of GGEs was confirmed also experimentally in a cold-atom setup [26] where, up to some time, a closed and integrable system can be prepared. Reference [26] showed that GGEs for a Lieb-Liniger model can provide an accurate description of an interacting trapped one-dimensional (1D) Bose gas. However, it is very difficult to simulate integrable systems due to their fine-tuned nature: adding practically any other terms to an integrable model will break integrability and cause eventual thermalization [27-36]. Therefore it was believed that in realistic systems traces of integrability can be seen only in the transient dynamics [37-46] while the steady state is always thermal due to realistic integrability breaking terms.

In recent works [47-49], Lange and Lenarčič demonstrated that properties related to integrability are not as fragile as previously believed: if one weakly drives an only approximately integrable system and at the same time allows it to cool via a weak coupling to the environment, the system will nonetheless relax to a steady state approximated with a generalized Gibbs ensemble, supplemented with a small correction $\delta \rho$ :

$$
\rho_{\infty} \equiv \lim _{t \rightarrow \infty} \rho(t)=\rho_{\mathrm{GGE}}+\delta \rho .
$$

The major difference to the closed strictly integrable setup, Eq. (3), is that here the Lagrange multipliers $\lambda_{i}$ are determined by the integrability breaking perturbations themselves, through a stationarity condition [47-49]:

$$
\partial_{t}\left\langle C_{k}\right\rangle \approx \operatorname{tr}\left[C_{k} \mathcal{L}_{p} \frac{e^{-\sum_{i} \lambda_{i} C_{i}}}{\operatorname{tr}\left[e^{-\sum_{i} \lambda_{i} C_{i}}\right]}\right] \stackrel{!}{=} 0, \quad \forall k .
$$

Here $\mathcal{L}_{p}$ denotes the Liouville operator corresponding to perturbations which weakly break the integrability, and as a consequence the conservation laws, while driving and cooling the system. Such a setup is much more versatile because it does not require the fine-tuned perfect integrability and at the same time allows for the engineering of GGEs through a particular choice of perturbations. A remarkable consequence is that one can stabilize steady states with large expectation values of nearly conserved operators $\left\langle C_{i}\right\rangle$, if a large corresponding $\lambda_{i}$ is established. For example, in solid-state spin chain materials, approximately described by an XXZ model coupled to phonons, a weak laser driving could stabilize steady states with huge heat and spin currents, since these are (partial) conservation laws of the XXZ model [47]. Alternatively, driving and openness can be provided by Markovian dissipative processes [48]. As shown in Fig. 1, the latter could be experimentally realized with trapped-ion platforms where long-range interaction between ions inevitably breaks integrability. By adding engineered dissipative processes, a GGE ensemble would be stabilized. As a consequence, the distribution $p\left(E_{n}\right)=\exp \left(-\sum_{i} \lambda_{i}\left\langle n\left|C_{i}\right| n\right\rangle\right) / \operatorname{tr}\left[e^{-\sum_{i} \lambda_{i} C_{i}}\right]$ over the eigenstates of $H_{0}|n\rangle=E_{n}|n\rangle$ would differ from a thermal one $p\left(E_{n}\right)=\exp \left(-\beta E_{n}\right) / \operatorname{tr}\left[e^{-\beta H_{0}}\right]$. While $p\left(E_{n}\right)$ is essentially impossible to measure experimentally, the nonthermal nature of $\rho_{\mathrm{GGE}}$ is more easily detected through possible exceedingly large expectation values of conserved operators $\left\langle C_{i}\right\rangle$ of parent $\mathrm{XY}$ or transverse-Ising Hamiltonians.

In this paper, we address the latter option and discuss an implementation based on ion-trap technology [50-52]. Controllable coherent couplings in ion-trap systems have been widely used for the realization of spin models [53,54], and numerous milestone experiments have been conducted on a variety of systems [55-63]. State-of-the-art Paul traps [51,59,62], Penning traps $[61,64,65]$, and microtraps [66-70] offer a rich toolbox of couplings suitable to engineer coherent Hamiltonians, as well as dissipative interactions. Sympathetic cooling based on mixed-species ion chains is well studied in Paul traps [71-73] where it is used to remove entropy from the motional modes of the ions. Here, we realize a driven-dissipative dynamics consisting of a spin Hamiltonian, in combination with one- and two-body dissipation. The dissipators are engineered combining tunable carrier and sideband couplings with repumper drives or sympathetic cooling as sources of dissipation. To tightly confine the motional modes to the interacting particles, we propose a mixed-species mode engineering technique that can be realized in microtrap arrays. The resulting dynamics stabilizes a steady state approximately described by a generalized Gibbs ensemble, despite different integrability breaking terms.

The paper is organized the following way: In Sec. II we introduce one choice of a Hamiltonian and Lindblad operators that could be realized in a trapped-ion experiment. In Sec. III we present numerical results and discuss experimental signatures and means to measure that a GGE approximates the stabilized steady state. In Secs. IV and V we present the engineering of the elementary dissipators. We then scale up the interactions in Secs. VI and VII, where we discuss the mode engineering in arrays of microtraps.

\section{MODEL}

The theory of activating integrability and engineering steady states described by generalized Gibbs ensembles in realistic systems is generic and applies to different systems approximately described by an integrable model, e.g., transverse-field Ising or XXZ Heisenberg chains, or LiebLiniger or Tonks-Girardeau Bose 1D gas. We choose an integrable model that is closest to the state-of-the art trappedion setups. We consider the XY Hamiltonian in the presence of a magnetic field $h$ :

$$
H_{\mathrm{XY}}=\sum_{j} J_{x} \sigma_{j}^{x} \sigma_{j+1}^{x}+J_{y} \sigma_{j}^{y} \sigma_{j+1}^{y}+h \sigma_{j}^{z},
$$

which belongs to the class of noninteracting integrable models. Such a Hamiltonian can be implemented by using standard techniques developed in the trapped-ion field. In contrast to Ref. [59], we rotate the spin axes by $\pi / 2$ around the $y$ axis. The resulting YZ model will allow us to facilitate an 
experimental implementation of Lindblad terms. We consider

$$
H_{0}=\sum_{j} J_{z} \sigma_{j}^{z} \sigma_{j+1}^{z}+J_{y} \sigma_{j}^{y} \sigma_{j+1}^{y}+h \sigma_{j}^{x} .
$$

An alternative realization based on the XY Hamiltonian in combination with sympathetic cooling is presented in Sec. V.

In traditional setups with trapped ions, the coupling between spins that are $d$ sites apart actually decays as $d^{-\alpha} \sigma_{j}^{z(y)} \sigma_{j+d}^{z(y)}$ with $\alpha \in[2,3]$. This is one inevitable source of integrability breaking since such Hamiltonian can no longer be diagonalized via a Jordan-Wigner transformation, neither is it Bethe-Ansatz solvable. However, if the decay is fast enough one can consider such a system as nearly integrable. In our analysis we will take into consideration only the leading contribution:

$$
H_{1}=\epsilon_{1} \sum_{j} J_{z} \sigma_{j}^{z} \sigma_{j+2}^{z}+J_{y} \sigma_{j}^{y} \sigma_{j+2}^{y}, \quad \epsilon_{1}=\frac{1}{2^{\alpha}} .
$$

The presence of $H_{1}$ alone would imply thermalization, i.e., relaxation to thermal expectation values of local observables, following a quench in such a system.

However, nonthermal steady states approximated by GGEs can be stabilized when weak couplings to Lindblad nonequilibrium baths are added. Nonthermal steady states are in principle expected for any non-Hermitian Lindblad operators. We empirically observe that the importance of additional conservation laws in the GGE, and thus the nonthermal nature, is more apparent for Lindblad operators with larger support. Couplings to more structured baths are also necessary to break symmetries that could prevent buildup of finite $\operatorname{tr}\left[C_{i} \rho\right]$. However, more structured Lindblad operators with larger support require more resources to be realized in a trapped-ion experiment. For that reason, we will consider the homogeneous bulk dissipators of two types, $a=1,2$,

$$
\mathcal{D}^{(a)} \rho=\sum_{j} L_{j}^{(a)} \rho L_{j}^{(a)^{\dagger}}-\frac{1}{2}\left\{L_{j}^{(a)^{\dagger}} L_{j}^{(a)}, \rho\right\}
$$

with Lindblad operators with support of one and two sites:

$$
\begin{gathered}
L_{j}^{(1)}=\sqrt{\epsilon(1-\gamma)} S_{j}^{-}, \quad \gamma \in[0,1], \\
L_{j}^{(2)}=\sqrt{\epsilon \gamma} S_{j}^{+} P_{j+1}^{\downarrow} .
\end{gathered}
$$

Here, $P_{j}^{\downarrow}=\frac{1}{2}\left(\mathbb{1}_{j}-\sigma_{j}^{z}\right)$ is a projection on the spin-down state at site $j$. The parameter $\epsilon$ gives the absolute strength of the coupling to baths, while $0 \leqslant \gamma \leqslant 1$ gives the relative strength of the two dissipators.

As we show below, the operators $L_{j}^{(2)}$ with support 2 are sufficiently structured to stabilize clearly nonthermal states approximated by GGEs and at the same time require a manageable amount of resources to be realized on trapped-ion platforms, as discussed in Secs. IV-VII. Even though the mechanism for stabilization of GGEs due to the coupling to nonthermal baths is rather generic, some Lindblad operators have a more visible effect than others: combining the chosen dissipators with $H_{\mathrm{XY}}$, Eq. (6), gives much weaker evidence of GGEs as compared to $H_{0}$, Eq. (7). While the $L_{j}^{(2)}$ operators are sufficient to observe the phenomenon we are interested in, having two types of couplings to baths with a relative

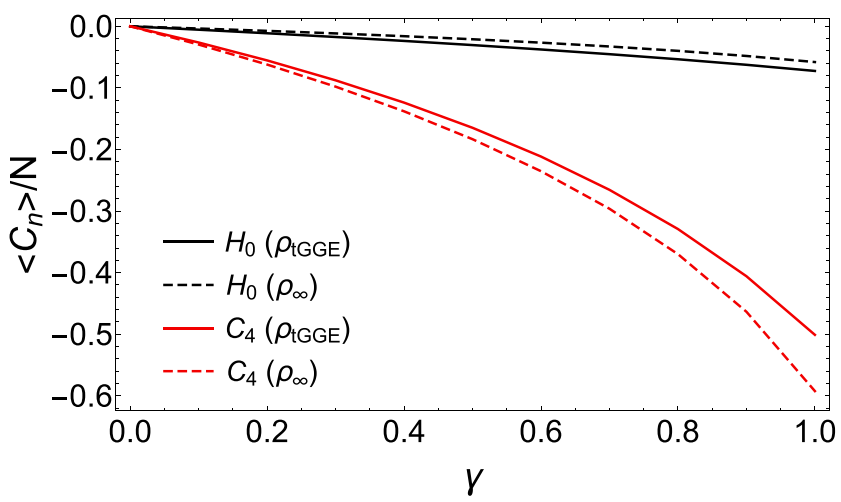

FIG. 2. $\left\langle H_{0}\right\rangle$ and $\left\langle C_{4}\right\rangle$ as a function of relative driving strength $\gamma$, Eqs. (10) and (11). A qualitative agreement between the exact $\rho_{\infty}$ and $\rho_{\mathrm{tGGE}}$ confirms that a generalized Gibbs ensemble is stabilized. A large $\left\langle C_{4}\right\rangle$, which could not be obtained from a nearly infinite temperature steady state consistent with a small $\left\langle H_{0}\right\rangle$, indicates that a highly nonthermal GGE is stabilized. Parameters: $J_{y}=h=1, J_{z}=$ $0.1 ; N=10, N_{c}=4$ for $\rho_{\mathrm{tGGE}}$ and $N=6, \epsilon=0.01$ for $\rho_{\infty}$.

strength $\gamma$ opens up additional possibilities for analysis and comparison with theory. Realization of $L_{j}^{(1)}$ is simple enough and thus practical for that matter.

The steady-state density matrix $\rho_{\infty}$ is determined by

$$
\dot{\rho}_{\infty}=\left(\mathcal{L}_{0}+\mathcal{L}_{p}\right) \rho_{\infty}=0,
$$

where $\mathcal{L}_{0}$ is a dominant term in the Liouvillian, while $\mathcal{L}_{p}=$ $\mathcal{L}_{u}+\mathcal{L}_{m}$ captures unitary and Markovian perturbations:

$$
\begin{aligned}
\mathcal{L}_{0} \rho & =-i\left[H_{0}, \rho\right], \quad \mathcal{L}_{u} \rho=-i\left[H_{1}, \rho\right], \\
\mathcal{L}_{m} \rho & =\left(\mathcal{D}^{(1)}+\mathcal{D}^{(2)}\right) \rho .
\end{aligned}
$$

Despite the fact that the underlying model $H_{0}$ in Eq. (6) is noninteracting, the next-nearest-neighbor interaction $H_{1}$ and our choice of Lindblad operators hinder analytical solvability and require a numerical solution. Finding exact solutions to weakly open, nearly integrable systems is generically a very hard problem [74,75]. Since our Lindblad operators are short ranged, we also cannot use the recently proposed hydrodynamic description [76].

For a review of the theory of weakly driven nearly integrable systems, developed in Refs. [47-49], see Appendix A. In the next section we go on to establish that this model does stabilize a steady state approximately described by a GGE ensemble, despite different sources of integrability breaking.

\section{EXPERIMENTAL SIGNATURES}

The experiment we are proposing would aim to show that a highly nonthermal steady state, described with a generalized Gibbs ensemble, can be stabilized in a nearly integrable model given by $H_{0}+H_{1}$, Eqs. (7) and (8), if weakly driven $(\epsilon \ll 1)$ with dissipation as in Eqs. (10) and (11).

To show that a GGE is stabilized by driving, we compare in Fig. 2 the steady-state expectation values obtained with the exact density matrix, Eq. (12), or with a GGE, Eq. (14). A qualitative agreement confirms that a GGE is stabilized. The first notable conclusion from the numerical analysis is that in the limit of small driving, $\epsilon \ll 1$, a truncated generalized 
Gibbs ensemble (tGGE)

$$
\rho_{\mathrm{tGGE}}=\frac{e^{-\sum_{i=1}^{N_{c}} \lambda_{i} C_{i}}}{\operatorname{tr}\left[e^{-\sum_{i=1}^{N_{c}} \lambda_{i} C_{i}}\right]}
$$

parametrized with only $N_{c}=4$ Lagrange parameters can capture expectation values of local observables we are interested in, while other more complicated conservation laws can be neglected. In comparison, a full density matrix requires $4^{N}$ parameters in the system size $N$. This shows that a description in terms of truncated GGEs is extremely compact. For a more thorough comparison see Appendix A.

Another interesting observation is that the next-nearestneighbor coupling $H_{1}$, Eq. (8), does not have a strong impact on the steady state. While in a closed setup $H_{1}$ is crucial as it dictates relaxation towards thermal expectation values, in our setup this is dominated by Lindblad terms (see Appendix A for details). Therefore, we neglect it in the results presented in the main text.

A physically most interesting consequence of stabilizing a steady state approximately described with a GGE is that the expectation values of conservation laws,

$$
\left\langle C_{k}\right\rangle=\operatorname{tr}\left[C_{k} \frac{e^{-\sum_{i} \lambda_{i} C_{i}}}{\operatorname{tr}\left[e^{-\sum_{i} \lambda_{i} C_{i}}\right]}\right],
$$

can be much larger than in a thermal state, $\operatorname{tr}\left[C_{k} e^{-\beta H_{0}} / \operatorname{tr}\left[e^{-\beta H_{0}}\right]\right]$. In this respect conservation laws are measurably distinct from other operators: a generic observable $O$ will have a much smaller expectation value than a conservation law, $\operatorname{tr}\left[O \rho_{\mathrm{GGE}}\right] \ll \operatorname{tr}\left[C_{k} \rho_{\mathrm{GGE}}\right]$, given they have the same norm $\operatorname{tr}\left[O^{2}\right]=\operatorname{tr}\left[C_{k}^{2}\right]$. The conservation law $C_{k}$ will show a particularly large expectation value if the driving is such that it stabilizes a GGE with a large corresponding Lagrange parameter $\lambda_{k}$. Which $\lambda_{k} \neq 0$ depends on the symmetry of the driving, i.e., the Lindblad operators $[47,48,77]$. A strong response of conservation laws to weak driving could have practical implications, such as heat and spin pumping $[47,48,77]$ in spin chain materials, but can also serve to detect that a GGE has been stabilized despite the integrability breaking terms. In the following we discuss ways to detect large expectation values of conserved (or partially conserved) operators, which could not be possible in a thermal state.

One possibility is to compare expectation values of observables which do or do not overlap with conservation laws. If driving stabilizes $\rho_{\mathrm{GGE}}$ with a large Lagrange multiplier $\lambda_{i}$ associated with the conservation law $C_{i}$, an observable $O$ that has a nonzero overlap with $C_{i}, \operatorname{tr}\left[O C_{i}\right] \neq 0$, will show a large expectation value. Let us, for example, consider $\left\langle\sigma_{j-1}^{y} \sigma_{j}^{x} \sigma_{j+1}^{y}\right\rangle \equiv\langle\mathrm{YXY}\rangle$ and $\left\langle\sigma_{j-1}^{y} \sigma_{j}^{y} \sigma_{j+1}^{x}\right\rangle \equiv\langle\mathrm{YYX}\rangle$. At least at small $J_{z} / J_{y} \ll 1$, where $H_{0} \approx J_{y} \sigma_{j}^{y} \sigma_{j+1}^{y}+h \sigma_{j}^{x}$, it is easy to estimate thermal expectation values $\langle\mathrm{XYX}\rangle_{\text {th }},\langle\mathrm{XXY}\rangle_{\text {th }}$ via expansion $\rho_{\text {th }}=\exp \left(-\beta H_{0}\right) / Z \approx\left(\mathbb{1}-\beta H_{0}+\beta^{2} H_{0}^{2} / 2+\right.$ $\ldots) / Z$ : a nonzero $\langle\mathrm{YYX}\rangle_{\text {th }}$ is dominantly coming from the second order in $\beta$, while $\langle\mathrm{YXY}\rangle_{\text {th }}$ from the third order. In a thermal state, $\left|\langle Y X Y\rangle_{\text {th }}\right| \ll\left|\langle Y Y X\rangle_{\text {th }}\right|$, as confirmed by a direct evaluation of thermal expectation values within a window of inverse temperatures $\beta$ with $\left|\langle Y Y X\rangle_{\text {th }}\right|$ comparable to the actual steady-state values $\langle$ YYX $\rangle$, Fig. 3(b).
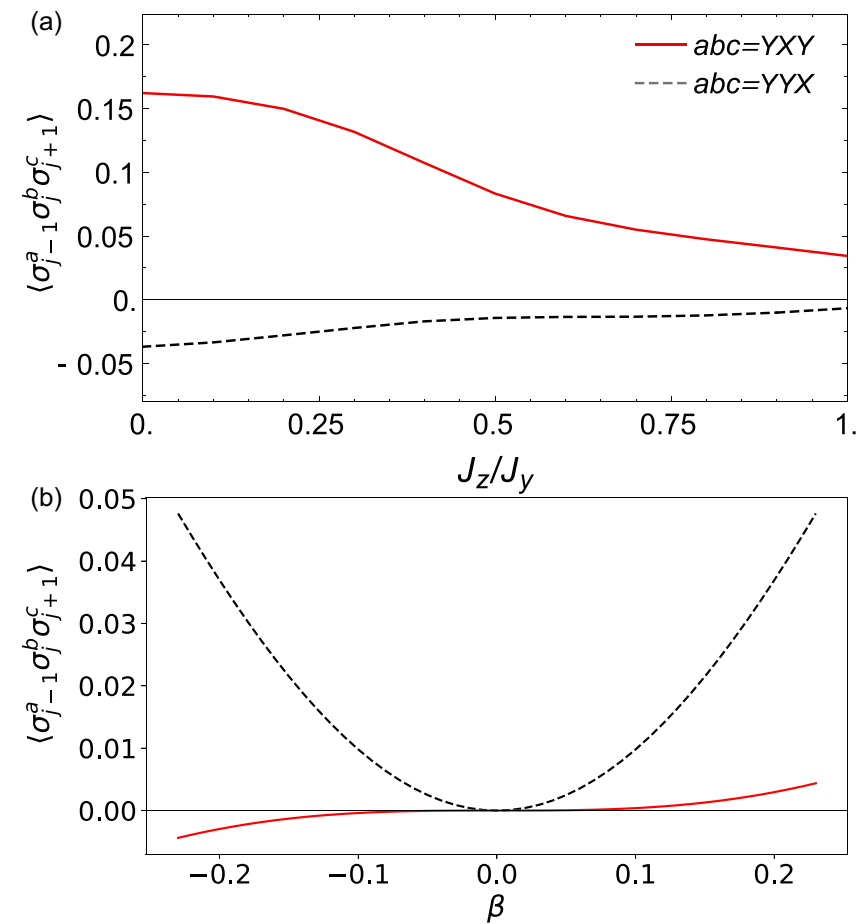

FIG. 3. (a) Steady-state expectation values $\left\langle\sigma_{j-1}^{y} \sigma_{j}^{y} \sigma_{j+1}^{x}\right\rangle$ and $\left\langle\sigma_{j-1}^{y} \sigma_{j}^{x} \sigma_{j+1}^{y}\right\rangle$ as a function of anisotropy $J_{z} / J_{y}$ obtained from the exact $\rho_{\infty}$ at $N=6, \epsilon=0.01, J_{y}=1, \gamma=0.8 .\left\langle\sigma_{j-1}^{y} \sigma_{j}^{x} \sigma_{j+1}^{y}\right\rangle$ shows a much larger value because it is part of a conservation law, $C_{4}$, Eq. (16). (b) Thermal expectation values for a range of inverse temperatures $\beta$, where $\left|\left\langle\sigma_{j-1}^{y} \sigma_{j}^{y} \sigma_{j+1}^{x}\right\rangle_{\text {th }}\right|$ is comparable to (a), are inconsistent with steady-state results because $\left\langle\sigma_{j-1}^{y} \sigma_{j}^{y} \sigma_{j+1}^{x}\right\rangle_{\text {th }}>0$ and $\left|\left\langle\sigma_{j-1}^{y} \sigma_{j}^{y} \sigma_{j+1}^{x}\right\rangle_{\mathrm{th}}\right| \gg\left|\left\langle\sigma_{j-1}^{y} \sigma_{j}^{x} \sigma_{j+1}^{y}\right\rangle_{\mathrm{th}}\right|$. Here $N=10, J_{y}=1, J_{z}=0.1$, $h=1$.

However, the true steady-state values in Fig. 3(a) show $|\langle\mathrm{YXY}\rangle| \gg|\langle\mathrm{YYX}\rangle|$. Besides, also the sign $\langle\mathrm{YYX}\rangle<0$ is inconsistent with a thermal state. These easily measurable observations are a clear sign that the steady state is not thermal. The large expectation value of $\langle\mathrm{YXY}\rangle$ is a direct consequence of the fact that this operator is part of $C_{4}$, Eq. (16), therefore its expectation value has also a linear contribution in $\lambda_{4}$ in the GGE expansion, since driving stabilizes a GGE with $\lambda_{4} \neq 0$.

In our setup, besides the Hamiltonian $H_{0}\left(H_{0}=C_{2}\right.$ according to the notation we use, see Appendix A), the next simplest local extensive conservation law $C_{i}$ that shows a strong response to the dissipative driving is

$$
\begin{aligned}
C_{4}= & \sum_{j} \sum_{\mu=z, y} J_{\mu} \sigma_{j}^{\mu} \sigma_{j+1}^{x} \sigma_{j+2}^{x} \sigma_{j+3}^{\mu} \\
& -h \sigma_{j}^{\mu} \sigma_{j+1}^{x} \sigma_{j+2}^{\mu}+J_{\bar{\mu}} \sigma_{j}^{\mu} \sigma_{j+1}^{\mu},
\end{aligned}
$$

where $\bar{z}=y$ and $\bar{y}=z$. Figure 2 shows the comparison of $\left\langle H_{0}\right\rangle=\left\langle C_{2}\right\rangle$ and $\left\langle C_{4}\right\rangle$ as a function of the relative strength $\gamma$, Eqs. (10) and (11), obtained from a GGE calculation on $N=10$ sites or a full steady-state density matrix on $N=6$ sites. While $\left|\left\langle H_{0}\right\rangle\right|(\gamma)$ shows rather small values, $\left|\left\langle C_{4}\right\rangle\right|(\gamma)$ is bigger. That would never be the case, if $\left\langle C_{4}\right\rangle(\gamma)$ was evaluated with respect to (an almost infinite temperature) thermal ensemble, which reproduces small $\left|\left\langle H_{0}\right\rangle\right|(\gamma)$. This observation alone suggests that a nonthermal steady state is stabilized. 


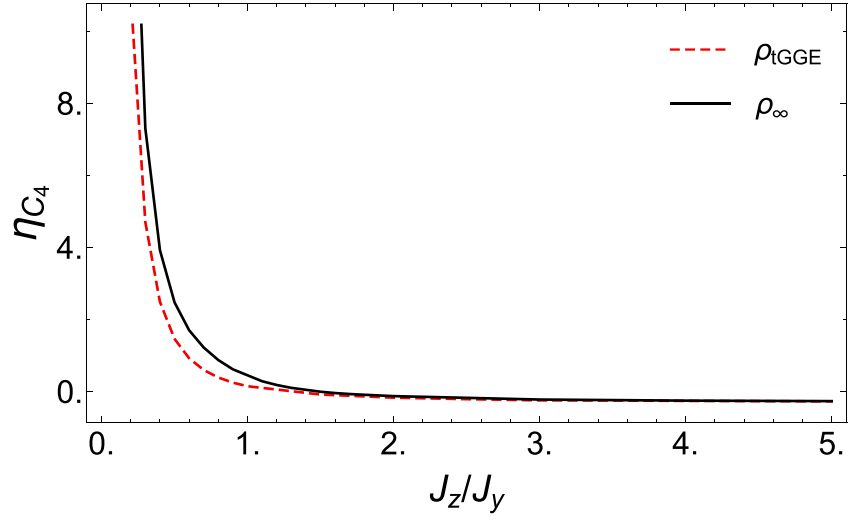

FIG. 4. The quantity $\eta_{C_{4}}$, Eq. (17), evaluated for $O=C_{4}$, measures how nonthermal the steady state is at different anisotropies $J_{z} / J_{y}$ for $J_{y}=h=1, \gamma=0.5$. It suggests that the experiment should optimally be performed at small $J_{z} / J_{y}$ or $J_{z}=0$. The calculation is based on the exact steady state $\rho_{\infty}$ at $\epsilon=0.01$ on $N=6$ sites and on the truncated GGE $\rho_{\mathrm{tGGE}}$ on $N=10$ sites using $N_{C}=4$ conservation laws.

In order to quantify how nonthermal the steady state is and to select the optimal parameters, we introduce $\eta_{O}$, which gives the relative deviation from the thermal expectation value for operator $O$,

$$
\eta_{O}=\frac{\operatorname{tr}\left[O \rho_{x}\right]-\operatorname{tr}\left[O \rho_{\mathrm{th}}\right]}{\operatorname{tr}\left[O \rho_{\mathrm{th}}\right]}
$$

calculated with respect to the exact steady state, $\rho_{x}=\rho_{\infty}$, or with the truncated GGE, $\rho_{x}=\rho_{\mathrm{tGGE}}$. For calculations with $\rho_{x}=\rho_{\infty}$ we define $\rho_{\text {th }}$ as a thermal state with respect to $H_{0}$, Eq. (6), with temperature determined from the condition $\operatorname{tr}\left[H_{0} \rho_{\infty}\right]=\operatorname{tr}\left[H_{0} e^{-\beta H_{0}} / \operatorname{tr}\left[e^{-\beta H_{0}}\right]\right]$. For calculations based on $\rho_{x}=\rho_{\mathrm{tGGE}}$ the temperature in $\rho_{\mathrm{th}}$ is calculated using a Gibbs ensemble ansatz with $H_{0}$ as the only conservation law.

In the following we focus on the operator $O=C_{4}$. In the absence of Lindblad driving, the system would thermalize due to the integrability breaking power-law decay of interactions between ions and one would measure thermal expectation values, i.e., $\eta_{C_{4}}=0$. In the presence of a weak Lindblad drive, on the other hand, the steady state can be highly nonthermal. Figure 4 shows $\eta_{C_{4}}$ as a function of anisotropy $J_{z} / J_{y}$, obtained from the exact density matrix $\rho_{\infty}$ on $N=6$ sites at $\epsilon=0.01$ and from a $\rho_{\mathrm{tGGE}}$ on $N=10$. The dependence on $J_{z} / J_{y}$ suggests that the experiment observing a highly nonthermal steady state $\left(\eta_{C_{4}} \gg 1\right)$ achieved by a weak driving should operate at a small $J_{z} / J_{y}$ or even at $J_{z}=0$ (corresponding to the transverse-field Ising case).

Why $\eta_{C_{4}}$ is so large for $J_{z}=0$ (and small $J_{z}$ ) can be reasoned by looking directly at the expectation values of $\left\langle H_{0}\right\rangle$ and $\left\langle C_{4}\right\rangle$, Fig. 5. $\left\langle H_{0}\right\rangle \approx 0$ is almost zero, suggesting an almost infinite temperature state $(\beta \approx 0)$, which would imply $\left\langle C_{4}\right\rangle \approx 0$. On the other hand, the observed $\left\langle C_{4}\right\rangle \sim 1$ is actually large. This observation, together with a good agreement between $\rho_{\infty}$ and $\rho_{\mathrm{tGGE}}$, clearly shows that a GGE with a large $\lambda_{4}$ is stabilized, despite $\beta \approx 0$. Measuring $\left\langle H_{0}\right\rangle$ and $\left\langle C_{4}\right\rangle$ would thus serve as strong affirmation of our theory.

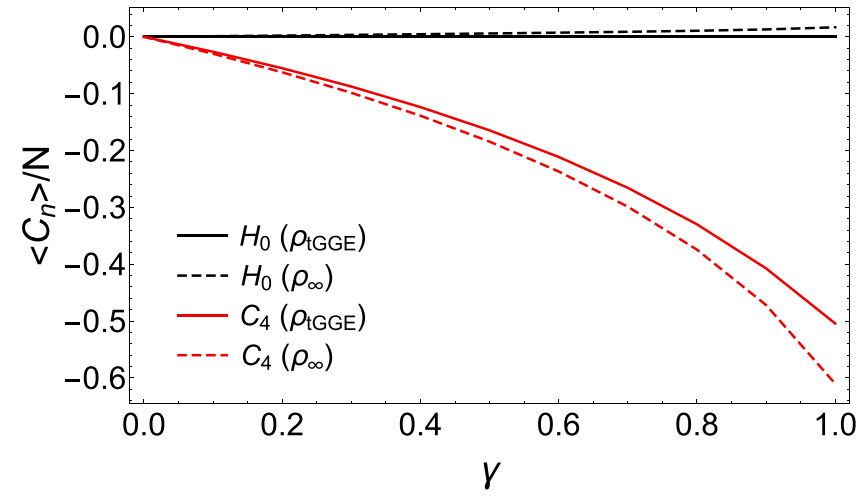

FIG. 5. Expectation values $\left\langle H_{0}\right\rangle$ and $\left\langle C_{4}\right\rangle$ as a function of relative driving strength $\gamma$, Eqs. (10) and (11), for $J_{z}=0$, corresponding to the transverse-field Ising case. The stabilized steady state is obviously highly nonthermal and described with a GGE with $\beta \approx 0$ and large $\lambda_{4}$, manifested via tiny $\left\langle H_{0}\right\rangle$ and large $\left\langle C_{4}\right\rangle$. Parameters: $J_{y}=h=1, J_{z}=0 ; N=10, N_{c}=4$ for $\rho_{\mathrm{tGGE}}$ and $N=6, \epsilon=0.01$ for $\rho_{\infty}$.

In Fig. 6, we show that at mild anisotropy, for example, $J_{z} / J_{y}=0.9$, also a magnetic field $h$ helps to prepare a more nonthermal state.

\section{DISSIPATION ENGINEERING}

Having shown that our dissipative driving can stabilize a steady state described by a GGE, we now discuss the implementation of the desired dynamics in a trapped-ion setup. To engineer suitable dissipative interactions, we combine coherent couplings with sources of dissipation such as induced spontaneous emission and sympathetic cooling. We use these tools to tailor the desired one- and two-body jump operators and verify their action numerically.

In this section, we assume that the YZ Hamiltonian in Eq. (7) is implemented. This allows us to engineer the dissipation in Eqs. (10) and (11) between the levels $|\uparrow\rangle,|\downarrow\rangle$. As these are eigenstates of (stimulated) spontaneous emission, it is possible to use repumper beams as sources of dissipation.

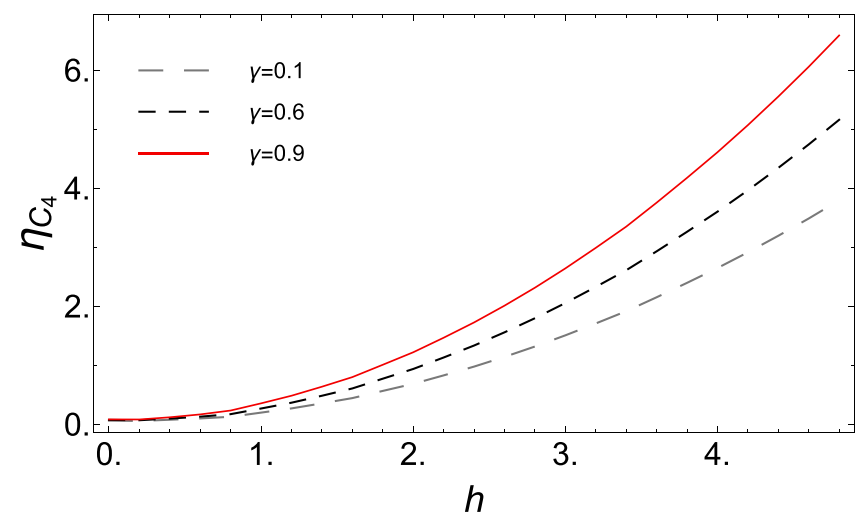

FIG. 6. Relative deviation from a thermal state $\eta_{C_{4}}$, Eq. (17), as a function of magnetic field $h$ for weak anisotropy $J_{z} / J_{y}=0.9$ obtained from tGGE at $N=10, J_{y}=1$ and different ratios of Lindblad drivings $\gamma=0.1,0.6,0.9$. Increasing the magnetic field assists in preparing a more nonthermal state. 
(a)
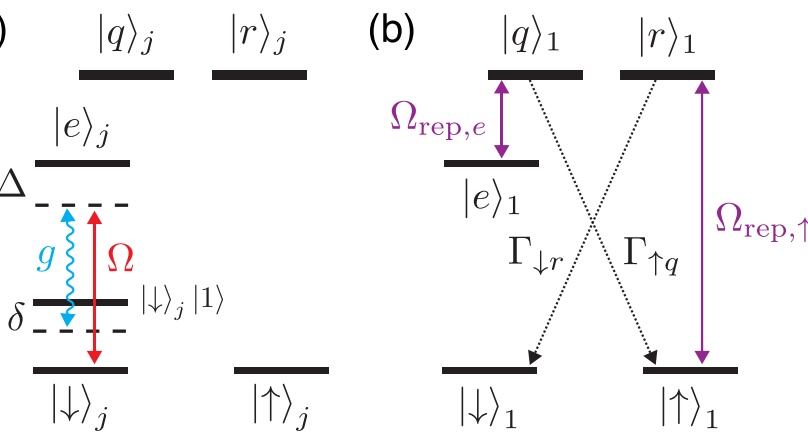

FIG. 7. Setup for engineered single-body and two-body dissipation. We consider two trapped ions, $j=1$ and 2 , with two stable ground levels, $|\downarrow\rangle$ and $|\uparrow\rangle$, and three excited levels, $|e\rangle,|r\rangle$, and $|q\rangle$. The ions are subject to coherent couplings (solid lines) and dissipation (dotted lines). (a) A weak drive excites both ions from $|\downarrow\rangle$ to $|e\rangle$ (strength $\Omega$, detuning $\Delta$ ). The transition from $|e\rangle$ to $|\downarrow\rangle$ is coupled to the motional mode $a$ (phonon detuning $\delta$ ) by a redsideband interaction (coupling constant $g$ ). The second ket denotes motional excitation and is dropped when in vacuum. (b) We realize single-body decay from $|\uparrow\rangle$ to $|\downarrow\rangle$ and from $|e\rangle$ to $|\uparrow\rangle$ on ion 1. This is done by optical pumping using tunable repumper beams (Rabi rates $\Omega_{\mathrm{rep}, \uparrow}$ and $\Omega_{\mathrm{rep}, e}$ ), via the unstable levels $|r\rangle$ and $|q\rangle$ (decay rates $\Gamma_{\downarrow r}$ and $\Gamma_{\uparrow q}$ ).

In Sec. V, in turn, we assume that the XY model in Eq. (6) is realized. This requires the dissipation to be engineered between the eigenstates of $\sigma_{x}$, as is demonstrated using sympathetic cooling.

\section{A. Setup}

To implement the one- and two-body jump operators in Eqs. (10) and (11), we consider a system of trapped ions coupled through motional modes. To simplify the discussion, we start by considering a minimal instance consisting of two ions indexed 1 and 2, and a motional mode $a$, and generalize to more ions in Secs. VI and VII. As is shown in Fig. 7, each of the ions is assumed to have two stable ground levels, $|\uparrow\rangle$ and $|\downarrow\rangle$, and three excited levels, $|e\rangle,|r\rangle$, and $|q\rangle$. The motional mode $a$ is assumed to be cooled to the ground state, $|0\rangle$. The free Hamiltonian of this system is given by

$$
H_{\text {free }}=\delta a^{\dagger} a+\sum_{j=1}^{2} \Delta|e\rangle_{j}\left\langle e\left|+\Delta_{r}\right| r\right\rangle_{j}\left\langle r\left|+\Delta_{q}\right| q\right\rangle_{j}\langle q| .
$$

Here we introduce a phonon detuning $\delta$ and an ionic detuning $\Delta$, assuming that we work in a suitable rotating frame with respect to the fields to be introduced below. We will use level $|r\rangle$ to realize the single-body decay in Eq. (10) and levels $|e\rangle$ and $|q\rangle$ in combination with mode $a$ for the two-body dissipation in Eq. (11). Level $|q\rangle$ will be employed to add a dissipation channel from level $|e\rangle$ to level $|\uparrow\rangle$. The ions are excited from $|\downarrow\rangle$ to $|e\rangle$ by a weak "carrier" drive

$$
H_{\text {drive }}=\frac{\Omega}{2} \sum_{j=1}^{2}|e\rangle_{j}\langle\downarrow|+\text { H.c. },
$$

with a Rabi frequency $\Omega$. In addition, levels $|\uparrow\rangle$ and $|e\rangle$ of ion 1 are excited to $|r\rangle$ and $|q\rangle$ by coherent "repumper" beams,

$$
\begin{aligned}
& H_{\text {rep }, \uparrow}=\frac{\Omega_{\text {rep }, \uparrow}}{2}|r\rangle_{1}\langle\uparrow|+\text { H.c. }, \\
& H_{\text {rep }, e}=\frac{\Omega_{\text {rep }, e}}{2}|q\rangle_{1}\langle e|+\text { H.c. },
\end{aligned}
$$

with Rabi rates $\Omega_{\text {rep, } \uparrow / e}$. The coupling between ions 1 and 2 , needed to engineer two-body dissipation, is mediated by a common motional mode, with creation (annihilation) operator $a^{\dagger}(a)$. This phonon mode is coupled to the transition from $|e\rangle$ to $|\downarrow\rangle$ by the red-sideband interaction

$$
H_{\text {int }}=g \sum_{j=1}^{2} a^{\dagger}|\downarrow\rangle_{j}\langle e|+a| e\rangle_{j}\langle\downarrow|+\text { H.c. },
$$

with a coupling constant $g$.

In addition to the above coherent interactions, the excited level $|r\rangle$ is assumed to be inherently unstable and to decay to level $|\downarrow\rangle$ by spontaneous emission, which can be described using the jump operators

$$
L_{\downarrow r, j}=\sqrt{\Gamma_{\downarrow r}}|\downarrow\rangle_{j}\langle r|, \quad(j=1,2) .
$$

The excited level $|q\rangle$, in turn, is assumed to decay to $|\uparrow\rangle$ :

$$
L_{\uparrow q, j}=\sqrt{\Gamma_{\uparrow q}}|\uparrow\rangle_{j}\langle q|, \quad(j=1,2) .
$$

To describe the joint dynamics of the ions and the phonons, we use the following notation: the state of the system is described by two kets, where the first ket denotes the internal state of the ions, e.g., $|\downarrow \downarrow\rangle=|\downarrow\rangle_{1}|\downarrow\rangle_{2}$. Motional excitations are indicated by a second ket, e.g., $|\downarrow \downarrow\rangle|1\rangle$, which is dropped when the motion is in the ground state.

\section{B. Single-body dissipation}

To realize single-body dissipation, we employ standard optical pumping, combining excitation from $|\uparrow\rangle$ to $|r\rangle$ by $H_{\text {rep }, \uparrow}$, Eq. (20), and decay from $|r\rangle$ to $|\downarrow\rangle$ by spontaneous emission $L_{\downarrow r, j}$. The effective jump operator [78] for the decay of level $|\uparrow\rangle$ to $|\downarrow\rangle$ through $|r\rangle$ is thus, after elimination of level $|r\rangle$, given by

$$
L_{\text {eff }}^{(1)}=\sqrt{\gamma_{1}}|\downarrow\rangle_{1}\left\langle\uparrow\left|\equiv \sqrt{\frac{\Omega_{\mathrm{rep}, \uparrow}^{2}}{\Gamma_{\downarrow r}}}\right| \downarrow\right\rangle_{1}\langle\uparrow| .
$$

We thereby realize the desired jump operator $L_{1}^{(1)}=$ $\sqrt{\epsilon(1-\gamma)} S_{1}^{-}$in Eq. (10). Using individual addressing techniques, this process can be made site specific. The decay rate $\gamma_{1}$ can be tuned by varying $\Omega_{\mathrm{rep}, \uparrow}$, assuming it to be much smaller than the natural linewidth of level $|r\rangle, \Gamma_{\downarrow r} \gg \Omega_{\text {rep }, \uparrow}$. Note that, while here we have only assumed the desired dissipation channel from $|r\rangle$ to $|\downarrow\rangle$, additional decay processes from these levels could be described by the same method.

To engineer the two-body dissipation in Eq. (11) in Sec. IV C below, we will also rely on an induced spontaneous emission process from $|e\rangle$ to $|\uparrow\rangle$. Here we assume that we can realize the YZ Hamiltonian in Eq. (7). Alternatively, in the presence of the XY Hamiltonian in Eq. (6), sympathetic cooling can be used as a source of dissipation, as is described in Sec. V. 
To realize optical pumping from $|e\rangle$ to $|\uparrow\rangle$, we couple $|e\rangle$ of ion 1 to the unstable level $|q\rangle$ using the repumper in Eq. (21). Together with the decay from $|q\rangle$ to $|\uparrow\rangle$ in Eq. (24), this realizes

$$
L_{\uparrow e}=\sqrt{\Gamma}|\uparrow\rangle_{1}\left\langle e\left|\equiv \sqrt{\frac{\Omega_{\text {rep }, e}^{2}}{\Gamma_{\uparrow q}}}\right| \uparrow\right\rangle_{1}\langle e| .
$$

Again, the decay rate $\Gamma$ is tunable through the strength of the corresponding repumper beam $\Omega_{\text {rep }, e}$.

\section{Two-body dissipation}

We now turn to the two-body dissipation in Eq. (11). Compared to the single-body dissipation in the previous section, the operator $L_{j}^{(2)}$ is more complicated to engineer, but itself sufficient to realize a highly nonthermal GGE (see Fig. 2 at $\gamma=1$ ). For our minimal instance of two ions, the operator reads $L_{1}^{(2)}=\sqrt{\epsilon \gamma} S_{1}^{+} P_{2}^{\downarrow}=\sqrt{\epsilon \gamma}|\uparrow \downarrow\rangle\langle\downarrow \downarrow|$. The action of this operator can be understood as a raising on spin 1, $S_{1}^{+}=|\uparrow\rangle_{1}\langle\downarrow|$, conditioned on the state of spin 2 .

We will now engineer the desired two-body dissipation based on the assumptions of weak driving, $\Omega^{2} \ll\left\{\Gamma^{2}, g^{2}\right\}$, and strong coupling, $\Gamma^{2} \ll g^{2}$. In this regime, the ground state $|\downarrow \downarrow\rangle|0\rangle$ is weakly excited by $H_{\text {drive }}$ to the excited state $\left|\psi_{e}\right\rangle|0\rangle=\frac{1}{\sqrt{2}}(|e \downarrow\rangle+|\downarrow e\rangle)|0\rangle$, which is composed of a superposition of excitations of both ions. Further excitation to the double-excited state $|e e\rangle$ can be neglected, as we will see further down. We engineer the desired mechanism using the couplings of $\left|\psi_{e}\right\rangle|0\rangle$.

The ion-excited state $\left|\psi_{e}\right\rangle|0\rangle$ is coupled to the motionexcited state $|\downarrow \downarrow\rangle|1\rangle$ by the red-sideband coupling $H_{\text {int }}$. Due to constructive interference between the excitation of the two ions, the corresponding rate is given by $\sqrt{2} g$. The Hamiltonian for the coupled subspace is

$$
\begin{aligned}
H_{\mathrm{e}, \downarrow \downarrow}= & \Delta\left|\psi_{e}\right\rangle|0\rangle\left\langle 0\left|\left\langle\psi_{e}|+\delta| \downarrow \downarrow\right\rangle\right| 1\right\rangle\langle 1|\langle\downarrow \downarrow| \\
& +\sqrt{2} g\left(\left|\psi_{e}\right\rangle|0\rangle\langle 1|\langle\downarrow \downarrow|+| \downarrow \downarrow\rangle| 1\rangle\langle 0|\left\langle\psi_{e}\right|\right),
\end{aligned}
$$

and illustrated in Fig. 8(a). Based on the assumption of a weak drive compared to the rapid dynamics of the excited subspace, we make a separation of timescales and first regard $H_{\mathfrak{e}, \downarrow \downarrow}$ alone, without the drive.

Provided strong coupling, the excited states of the strongly coupled subspace hybridize and form dressed states $\left|\psi_{ \pm}\right\rangle$at detunings

$$
\Delta_{ \pm}=\frac{\Delta+\delta}{2} \pm \frac{1}{2} \sqrt{(\Delta+\delta)^{2}+4\left(\Delta \delta-2 g^{2}\right)} .
$$

Setting the ionic and the motional detunings to $\Delta \delta=2 g^{2}$ (e.g., $\Delta=\delta=\sqrt{2} g$ ) brings the lower dressed state in resonance with the drive $\Omega$, i.e., $\Delta_{-}=0$. As a consequence, $|\downarrow \downarrow\rangle$ is resonantly excited to $\left|\psi_{e}\right\rangle$ which in turn decays to $|\uparrow \downarrow\rangle$ at a rate $\Gamma$. This results in the required effective decay from $|\downarrow \downarrow\rangle$ to $|\uparrow \downarrow\rangle$, mediated by the resonant lower dressed state, $\left|\psi_{-}\right\rangle$. In Appendix B we present a full microscopic derivation that yields the two-body Lindblad operator, Eq. (11):

$$
L_{\text {eff }}^{(2)}=\sqrt{\gamma_{2}}|\uparrow \downarrow\rangle_{1,2}\langle\downarrow \downarrow| .
$$

(a) $|\downarrow \downarrow\rangle|1\rangle \sqrt{2} g\left|\psi_{e}\right\rangle|0\rangle=(|e \downarrow\rangle+|\downarrow e\rangle)|0\rangle / \sqrt{2}$
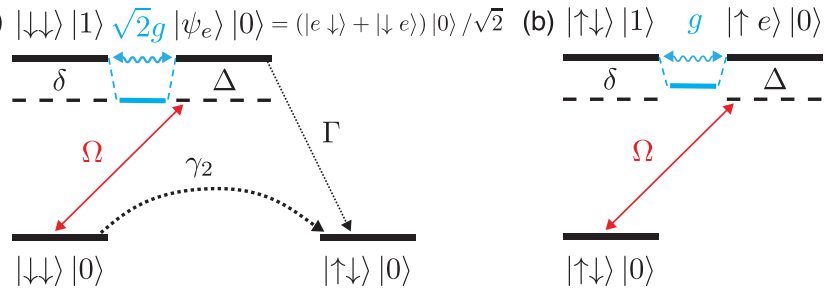

FIG. 8. Engineering of the two-body dissipation. (a) Desired effective decay process. State $|\downarrow \downarrow\rangle|0\rangle$ is coupled to the ion-excited state $\left|\psi_{e}\right\rangle|0\rangle=\frac{1}{\sqrt{2}}(|e \downarrow\rangle+|\downarrow e\rangle)|0\rangle$ by the drive $\Omega$. $\left|\psi_{e}\right\rangle|0\rangle$ is strongly coupled to the motion-excited state $|\downarrow \downarrow\rangle|1\rangle$ by the sideband coupling $g$, which is enhanced by a factor $\sqrt{2}$ due to constructive interference. While the bare states $\left|\psi_{e}\right\rangle|0\rangle$ and $|\downarrow \downarrow\rangle|1\rangle$ reside at detunings $\Delta$ and $\delta$, for $\Delta \delta=2 g^{2}$ their lower dressed state (indicated in blue) is in resonance with the drive and hence rapidly excited from $|\downarrow \downarrow\rangle|0\rangle$. Through its contribution from $\left|\psi_{e}\right\rangle|0\rangle$, it decays to $|\uparrow \downarrow\rangle|0\rangle$ by spontaneous emission $\Gamma$. These resonant couplings form an effective decay process from $|\downarrow \downarrow\rangle|0\rangle$ to $|\uparrow \downarrow\rangle|0\rangle$, at an enhanced rate $\gamma_{2}$. (b) Undesired process. Also state $|\uparrow \downarrow\rangle|0\rangle$ is excited by the drive, to an ion-excited state $|e \uparrow\rangle|0\rangle$. The sideband coupling couples $|e \uparrow\rangle|0\rangle$ to $|\uparrow \downarrow\rangle|1\rangle$ at a coupling constant $g$. Their dressed states are thus out of resonance and only weakly populated by the drive.

Using second-order perturbation theory in $\Omega / \Gamma$ the effective decay rate is given by

$$
\gamma_{2}=\frac{4 \Omega^{2}}{\Gamma} .
$$

The decay rate $\gamma_{2}$, and hence the relative strength of the singleand two-body dissipation, can thus be adjusted by varying $\Omega$ and $\Gamma\left(\Omega_{\text {rep }, e}\right) \cdot \gamma_{2}$ is ultimately limited by the linewidth $\Gamma_{q}$ of level $|q\rangle$, which is involved in constructing the engineered single-body decay in Eq. (26).

Compared to $\gamma_{2}$, effective decay processes mediated by $|e e\rangle$ can be neglected: $|e e\rangle$ forms a coupled two-excitation subspace with $\left|\psi_{e}\right\rangle|1\rangle$ and $|\downarrow \downarrow\rangle|2\rangle$ (couplings $\sqrt{2} g$ and $2 g$ ). While for the above parameter choice the drive from $\left|\psi_{e}\right\rangle$ is in resonance with the two-excitation dressed states, the coupling rates of the so mediated effective process only enter to fourth order in perturbation theory and are thus negligibly small. Also AC Stark shifts arising from the weak off-resonant excitation of $|\uparrow \downarrow\rangle$ (see Appendix C) can be safely ignored in the considered parameter regime.

Another imperfection inherent to the scheme is given by the population of the excited level $|\uparrow e\rangle$ which is offresonantly excited from $|\uparrow \downarrow\rangle$ by the drive $\Omega$, as illustrated in Fig. 8(b). For perfect individual addressing of the first ion by $H_{\text {rep }, e},|\uparrow e\rangle$ is steadily populated. Using adiabatic elimination, it is possible to estimate the steady-state population of an excited state such as $|\uparrow e\rangle[79,80]$, which scales

$$
P_{\uparrow e} \sim \frac{\Omega^{2}}{g^{2}} .
$$

Population of the excited state $|\uparrow e\rangle$ is thus expected to be largely suppressed, as we numerically confirm in the following.

We verify the engineering of the two-body dissipation and assess its performance numerically by simulating the 


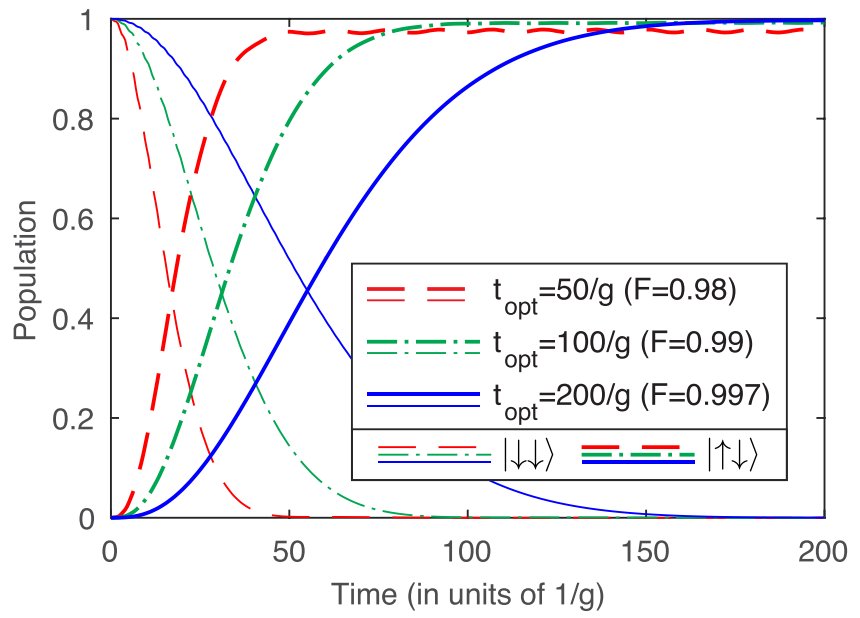

FIG. 9. Numerical simulation. We verify the engineering of the two-body dissipation by simulating the dynamics and plotting the population of the states $|\downarrow \downarrow\rangle$ (thin lines) and $|\uparrow \downarrow\rangle$ (thick lines) over time, starting from an initial state $|\downarrow \downarrow\rangle$. We present three different parameter choices, where we optimize the action of the scheme at different times $t_{\mathrm{opt}}=\{50,100,200\} / \mathrm{g}$ (dashed lines, dash-dotted lines, solid lines). We obtain fidelities $F_{\mathrm{opt}}=\{0.98,0.99,0.997\}$ for the parameter choices $\Gamma_{\mathrm{opt}}=\{0.82,0.48,0.29\} \mathrm{g}$ and $\Omega_{\mathrm{opt}}=$ $\{0.15,0.08,0.05\} g \approx \Gamma_{\text {opt }} / 6$. Population in the excited states is negligible.

dynamics given by the master equation

$$
\begin{aligned}
\dot{\rho} & =-i\left[H_{\text {free }}+H_{\text {drive }}+H_{\text {int }}, \rho\right]+\mathcal{D}^{(\uparrow e)} \rho, \\
\mathcal{D}^{(\uparrow e)} \rho & =L_{\uparrow e} \rho L_{\uparrow e}^{\dagger}-\frac{1}{2}\left\{L_{\uparrow e}^{\dagger} L_{\uparrow e}, \rho\right\} .
\end{aligned}
$$

We use this to obtain (i) the optimal parameter choice, (ii) the extent of unwanted population of the excited level $|\uparrow e\rangle$, and (iii) the timescales of the dissipative compared to the unitary dynamics.

We assume that the system starts from $|\downarrow \downarrow\rangle|0\rangle$ and optimize the fidelity of the state $|\uparrow \downarrow\rangle|0\rangle$ after a chosen time $t_{\text {opt }}=\{50,100,200\} / g$ by the choice of the parameters $\Gamma$ and $\Omega$. The result is plotted in Fig. 9. From the initial state $|\downarrow \downarrow\rangle$, the system evolves to fidelities with $|\uparrow \downarrow\rangle$ of $F_{\mathrm{opt}}=\{0.98,0.99,0.997\}$ for the parameter choices $\Gamma_{\mathrm{opt}}=$ $\{0.82,0.48,0.29\} g$ and $\Omega_{\text {opt }}=\{0.15,0.08,0.05\} g \approx \Gamma_{\text {opt }} / 6$. After a short nonexponential transient $\left(t<1 / \gamma_{\text {eff }}\right)$ which builds up the excited-state population, the curves attain the desired exponential form that is described by the effective jump operator in Eq. (29). This represents a close approximation to the desired dynamics.

The optimal parameters fulfill the conditions for weak driving and strong coupling, $\Omega^{2} \ll \Gamma^{2} \ll g^{2}$, which are the assumptions used in the above analysis. The residual population in $|\uparrow e\rangle$ is found to be $P_{\uparrow \mathrm{e}} \approx\{0.02,0.008,0.003\}$ and thus indeed negligible.

For the effective dissipation rate in Eq. (30) we obtain $\gamma_{2}=\{0.054,0.027,0.014\} g$. For typical values of $g /(2 \pi) \sim 10 \mathrm{kHz}$, this yields $\gamma_{2}=\{3.4,1.7,0.89\} / \mathrm{ms}$. The convergence time $\tau=1 / \gamma_{2}$ (corresponding to the decay of the initial-state population to $1 / e$ ) is found to be $\tau=\{18,37,70\} / g=\{0.29,0.59,1.1\} \mathrm{ms}$, in good agreement with the results plotted in Fig. 9.
The achievable values for $\gamma_{2}$ should be compared with the typical coupling strength in realizations of the spin models of about $J_{x / y} /(2 \pi) \sim 100 \mathrm{~Hz}[59,60]$, and the corresponding timescale of $\tau_{\text {spin }} \sim 1 \mathrm{~ms}$. From the above numbers it can be seen that the effective dissipation rate can be tuned to values within the same order of magnitude as the coupling constants of the spin model. Obtaining smaller values for $\gamma_{2}$-and thus making the dissipation into a perturbation as assumed in Secs. II and III-is in turn achieved by choosing weaker repump and driving rates, $\Gamma$ and $\Omega$.

\section{GENERALIZATION OF THE COUPLINGS TO THE $x$ BASIS}

Spin models along arbitrary directions, with and without anisotropy, can be realized on trapped-ion platforms, such as Paul traps and Penning traps, and also in microtraps [53]. The majority of the available setups, however, support XY Hamiltonians without anisotropy [59]. In the preceding section, we assumed the less common YZ spin Hamiltonian which enabled us to engineer the dissipation in the $z$ basis, the eigenbasis of decay by spontaneous emission. As an alternative to such implementation, we can utilize the more standard XY Hamiltonian in Eq. (6). We should note, however, that in order to stabilize a nontrivial $\left(\rho_{\infty} \neq \mathbb{1}\right)$ and distinguishingly nonthermal steady state an engineered form of Lindblad operators with proper symmetries must be used. For example, the XY Hamiltonian in combination with the dissipation in the $z$ basis would not result in a large $\left\langle C_{4}\right\rangle$. This problem is resolved by using the XY Hamiltonian in combination with dissipation in the rotated $x$ basis, the engineering of which is presented below.

We point out that a simpler alternative is given by using the XY Hamiltonian in combination with any type of Lindblad dissipation. Such setting would yield a nontrivial dynamics towards a possibly trivial steady state, where time evolution is approximately captured with a time-dependent GGE [48]. However, the nonthermal features might not be very pronounced in a generic setup, therefore we focus here on a setup with nontrivial steady states with clearly nonthermal nature.

We now engineer the dissipation in the $x$ direction which can stabilize a steady-state GGE,

$$
\begin{gathered}
L_{j}^{(1, x)}=\sqrt{\epsilon(1-\gamma)} S_{j, x}^{-}, \\
L_{j}^{(2, x)}=\sqrt{\epsilon \gamma} S_{j, x}^{+} P_{j+1, x}^{\downarrow},
\end{gathered}
$$

when combined with the XY Hamiltonian. Formally speaking, the change from the $z$ to the $x$ basis amounts to replacing the eigenstates of $\sigma_{z},\{|\downarrow\rangle,|\uparrow\rangle\}$ with the eigenstates of $\sigma_{x},| \pm\rangle=$ $(|\downarrow\rangle \pm|\uparrow\rangle) / \sqrt{2}$, i.e., $|\downarrow\rangle \mapsto|-\rangle,|\uparrow\rangle \mapsto|+\rangle$, in all steps of our previous derivation performed for the dissipation in the $z$ direction. Physically, using $| \pm\rangle$ is realized by coupling to both transitions $|\downarrow\rangle \leftrightarrow|e\rangle$ and $|\uparrow\rangle \leftrightarrow|e\rangle$ coherently. The resulting interactions, illustrated in Fig. 10(a), read

$$
\begin{gathered}
H_{\text {drive }, \mathrm{x}}=\frac{\Omega}{2} \sum_{j=1}^{2}|e\rangle_{j}\langle-|+\text { H.c., } \\
H_{\text {int }, \mathrm{x}}=g \sum_{j=1}^{2}\left(a^{\dagger}|-\rangle_{j}\langle e|+a| e\rangle_{j}\langle-|\right) .
\end{gathered}
$$


(a)

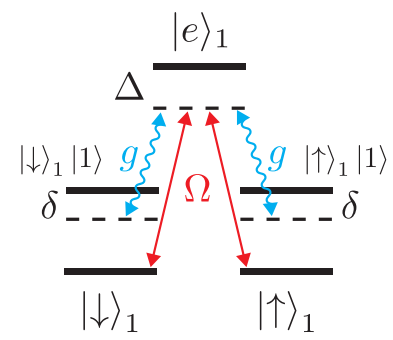

(b)

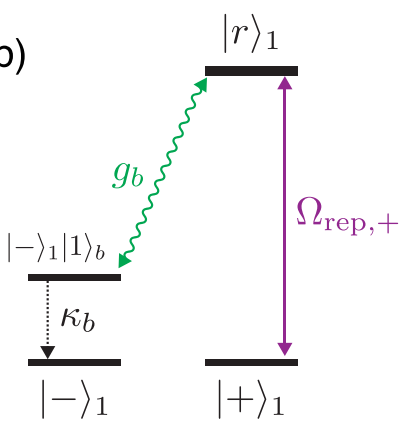

FIG. 10. Generalization of the couplings to the $x$ basis. (a) To engineer two-body dissipation in the $x$ basis, we use couplings between the states $| \pm\rangle=(|\downarrow\rangle \pm|\uparrow\rangle) / \sqrt{2}$, and $|e\rangle$. This is achieved by coupling the levels $|\downarrow\rangle$ and $|\uparrow\rangle$ coherently to $|e\rangle$. (b) Dissipation in the $x$ basis is facilitated by sympathetic cooling of the motion. The single-body decay process from $|+\rangle$ to $|-\rangle$ is engineered by excitation from $|+\rangle$ by a weak drive $\left(\Omega_{\mathrm{rep},+}\right)$ to an auxiliary level $|r\rangle$, a sideband coupling of the transition $|r\rangle \rightarrow|-\rangle$ to a motional mode $b\left(g_{b}\right)$, and sympathetic cooling of $b\left(\kappa_{b}\right)$. Decay from $|e\rangle$ to $|+\rangle$ (not shown) is engineered accordingly using a motional mode $c$, as is described in the text.

On the other hand, decay by spontaneous emission, as utilized in the previous sections, naturally occurs in the $z$ basis, $\{|\downarrow\rangle,|\uparrow\rangle\}$. In contrast to this, we now need to engineer sources of dissipation in the $x$ basis, which replaces Eqs. (23) and (26) in the $z$ basis. In the following, we demonstrate how to achieve this using decay of excitations via the motional degree of freedom by sympathetic cooling.

As shown in Fig. 10(b), to implement the single-body decay in Eq. (33), we excite $|+\rangle$ to the auxiliary level $|r\rangle$ by a repumper:

$$
H_{\text {rep },+}=\Omega_{\text {rep },+}|r\rangle_{1}\langle+|+\text { H.c. }
$$

The excitation to level $|r\rangle$ is transferred coherently to an auxiliary motional mode $b$ using a sideband interaction,

$$
H_{\mathrm{b}}=g_{\mathrm{b}} b^{\dagger}|-\rangle_{1}\langle r|+\text { H.c., }
$$

with a coupling constant $g_{\mathrm{b}}$. Mode $b$ is subject to sympathetic cooling which realizes the jump operator:

$$
L_{\mathrm{b}}=\sqrt{\kappa} b .
$$

Adiabatic elimination of $b$ leads, for $g_{b} \ll \kappa$, to the desired decay channel

$$
L_{-r}=\sqrt{\Gamma_{-r}}|-\rangle_{1}\langle r|,
$$

with a rate $\Gamma_{-r}=g_{b}^{2} / \kappa$, in analogy to Eq. (23). We realize the decay $L_{+e}=\sqrt{\Gamma_{+e}}|+\rangle_{1}\langle e|$ [see Eq. (26)] in a similar fashion, utilizing a motional mode $c$, which is subject to sympathetic cooling. Here, we couple $|e\rangle|0\rangle_{c}$ to $|+\rangle|1\rangle_{c}$ by a sideband drive $\left(g_{c}\right)$, which then decays to $|+\rangle|0\rangle_{c}$ by sympathetic cooling $\left(\kappa_{c}\right)$, resulting in an effective decay rate $\Gamma_{+e}=g_{c}^{2} / \kappa_{c}$. Involving level $|q\rangle$ is not necessary. Carrying out the same analysis as in Appendix B, we obtain the effective operator

$$
L_{\text {eff }}^{(2, x)}=\sqrt{\gamma_{2, \mathrm{x}}}|+-\rangle_{1,2}\langle--|,
$$

with a tunable decay rate $\gamma_{2, \mathrm{x}}=4 \Omega^{2} / \Gamma_{+e}^{2}$. We have thus realized the desired two-body dissipation in the $x$ basis in Eq. (34) by means of sympathetic cooling.

\section{SCALABLE IMPLEMENTATION}

Next, we discuss how to scale the mechanisms discussed in Secs. IV and V to larger numbers of ions. For a scalable implementation of our scheme, we assume a chain of $N$ ions (with even $N$ ) and a level structure similar to Sec. IV A. The physical system for the scalable implementation of two-body dissipation in Eq. (11) is shown in Fig. 11. The implementation of such system based on ion microtraps is presented in Sec. VII. Here we seek to implement interactions on all pairs of ions, such as $\{2 j-1,2 j\}$ and $\{2 j, 2 j+1\}$, as illustrated in Fig. 11(a). However, care has to be taken to avoid interference effects of the coherent couplings in the overlapping region, i.e., here ion $2 j$. We achieve this by devising two independent coupling configurations to mediate the engineered decay on the two different groups of ions, $\{2 j-1,2 j\}$ and $\{2 j, 2 j+$ $1\}$, as can be seen from Figs. 11(b) and 11(c). We assume each ion to have two (meta-) stable excited levels, $|e\rangle$ and $|f\rangle$, which are selectively addressable using, e.g., polarization selection rules.

For dissipation on pairs $\{2 j-1,2 j\}$, level $|e\rangle$ is used to mediate the two-body dissipation, whereas for pairs $\{2 j, 2 j+$ $1\}$ this is facilitated by level $|f\rangle$. Correspondingly, we employ two sets of localized phonon modes: Modes $a_{2 j-1,2 j}$ interact with ions $\{2 j-1,2 j\}$, and modes $a_{2 j, 2 j+1}$ couple to pairs $\{2 j, 2 j+1\}$. The engineering of the mode structure will be discussed in detail in Sec. VII.

To implement two-body dissipation, we again use tunable optical pumping of $|e\rangle$ and $|f\rangle$ to $|\uparrow\rangle$, in analogy to Sec. IV C, Eq. (26). Utilizing different individually addressed repumper beams for "odd" ions $2 j-1$ and "even" ions $2 j$, we realize

$$
\begin{aligned}
L_{\uparrow e, 2 j-1} & =\sqrt{\Gamma}|\uparrow\rangle_{2 j-1}\langle e|, \\
L_{\uparrow f, 2 j} & =\sqrt{\Gamma}|\uparrow\rangle_{2 j}\langle f| .
\end{aligned}
$$

Odd ions $2 j-1$ thus decay from $|e\rangle$ to $|\uparrow\rangle$, whereas even ions $2 j$ decay from $|f\rangle$ to $|\uparrow\rangle$, both at an equal rate $\Gamma$.

For the continuous and measurement-free interrogation of the system, we use two sets of coherent drives,

$$
\begin{gathered}
H_{\text {drive }}=H_{\text {drive }, e}+H_{\text {drive }, f}, \\
H_{\text {drive }, e}=\frac{\Omega}{2} \sum_{j=1}^{N / 2}\left(|e\rangle_{2 j-1}\langle\downarrow|+| e\rangle_{2 j}\langle\downarrow|\right)+\text { H.c., } \\
H_{\text {drive }, f}=\frac{\Omega}{2} \sum_{j=1}^{N / 2}\left(|f\rangle_{2 j}\langle\downarrow|+| f\rangle_{2 j+1}\langle\downarrow|\right)+\text { H.c., }
\end{gathered}
$$


(a)
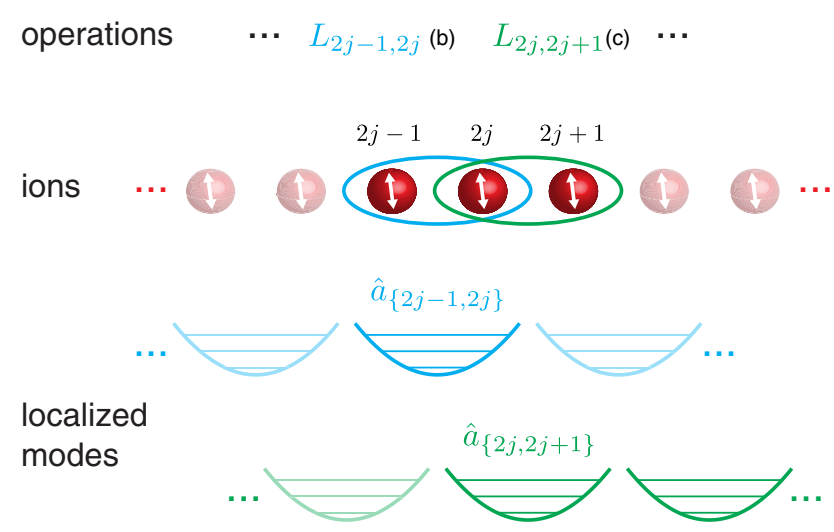

(b)

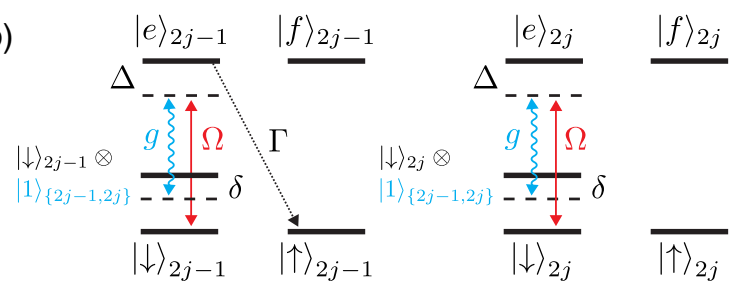

(c)

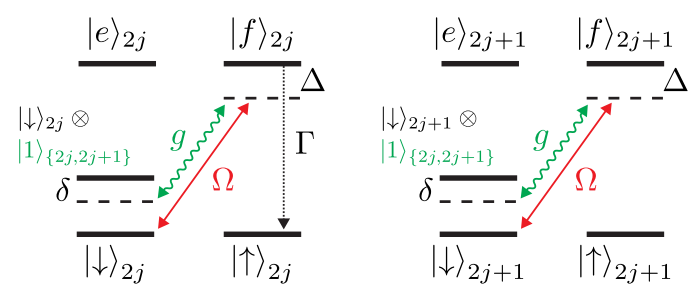

FIG. 11. Scalable implementation in trapped ions. (a) Setup. We assume a string of $N$ ions, denoted $2 j \pm 1$ for odd ions and $2 j$ for even ions. Two-body dissipation represented by the jump operators $L_{2 j-1,2 j}$ and $L_{2 j-1,2 j}$ is realized by coupling pairs of ions to localized phonon modes. Two sets of motional modes, $a_{2 j-1,2 j}$ and $a_{2 j, 2 j+1}$, couple to pairs of ions $\{2 j-1,2 j\}$ and $\{2 j, 2 j+1\}$. This avoids interference on the overlap ion $2 j$. (b, c) Coupling configurations for pairs of ions $\{2 j-1,2 j\}$ (b) and $\{2 j, 2 j+1\}$ (c). We assume two addressable excited levels, $|e\rangle$ and $|f\rangle$, for each ion. For odd ions $2 j-1$ (even ions $2 j$ ), we facilitate a decay from level $|e\rangle(|f\rangle$ ) to level $|\uparrow\rangle$ at a rate $\Gamma$, as is described in the text. The coherent drive $\Omega$ and the sideband couplings $g$ act on the transition from $|e\rangle$ to $|\downarrow\rangle$ (from $|f\rangle$ to $|\downarrow\rangle$ ) on pairs of ions $\{2 j-1,2 j\}$ $(\{2 j, 2 j+1\})$.

coupling the ground level $|\downarrow\rangle$ to the excited level $|e\rangle$ or $|f\rangle$, as well as sideband interactions:

$$
\begin{gathered}
H_{\text {int }}=H_{\text {int }, e}+H_{\text {int }, f}, \\
H_{\text {int }, e}=g \sum_{j=1}^{N / 2} a_{2 j-1,2 j}^{\dagger}\left(|\downarrow\rangle_{2 j-1}\langle e|+| \downarrow\rangle_{2 j}\langle e|\right)+\text { H.c., } \\
H_{\text {int }, f}=g \sum_{j=1}^{N / 2} a_{2 j, 2 j+1}^{\dagger}\left(|\downarrow\rangle_{2 j}\langle f|+| \downarrow\rangle_{2 j+1}\langle f|\right)+\text { H.c. }
\end{gathered}
$$

These realize coupling configurations, by which the transition $|e\rangle \leftrightarrow|\downarrow\rangle(|f\rangle \leftrightarrow|\downarrow\rangle)$ of any pair of ions $\{2 j-1,2 j\}(\{2 j-$ $1,2 j\})$ is coupled to a localized motional mode $a_{2 j-1,2 j}$ $\left(a_{2 j, 2 j-1}\right)$.

As a result, following the recipe in Sec. IV C, we realize jump operators acting on pairs of ions over the whole chain:

$$
\begin{gathered}
L_{2 j-1}^{(2)}=\sqrt{\gamma_{2}}|\uparrow \downarrow\rangle_{2 j-1,2 j}\langle\downarrow \downarrow|=\sqrt{\gamma_{2}} S_{2 j-1}^{+} P_{2 j}^{\downarrow}, \\
L_{2 j}^{(2)}=\sqrt{\gamma_{2}}|\uparrow \downarrow\rangle_{2 j, 2 j+1}\langle\downarrow \downarrow|=\sqrt{\gamma_{2}} S_{2 j}^{+} P_{2 j+1}^{\downarrow} .
\end{gathered}
$$

In the second step, these operators are brought back into the form of Eq. (11). Making the association $\gamma_{2}=\epsilon \gamma$, we have thereby engineered the desired two-body dissipation in a scalable manner.

Single-body dissipation in Eq. (10) is again realized-now for the whole chain-following the recipe in Sec. IV B: Using locally addressed repumper beams to an unstable level $|r\rangle$ for each individual ion, we achieve local jump operators:

$$
L_{j}^{(1)}=\sqrt{\gamma_{1}}|\downarrow\rangle_{j}\left\langle\uparrow\left|\equiv \sqrt{\frac{\Omega_{\mathrm{rep}, \uparrow}^{2}}{\Gamma_{\downarrow r}}}\right| \downarrow\right\rangle_{j}\langle\uparrow| .
$$

Associating $\gamma_{1}=\epsilon(1-\gamma)$, we have thus realized the desired single-body dissipation [Eq. (10)] for all ions in the chain.
The implementation of generalized dissipation in the $x$ basis, such as those in Eqs. (33) and (34) in Sec. V, can be scaled up in an analogous manner.

\section{NORMAL MODE ENGINEERING IN AN ARRAY OF MICROTRAPS}

In the following, we discuss the physical implementation of the scalable setting detailed in Sec. VI based on ion microtraps [66-70]. To implement the desired mode structure, with localized modes subject to dissipation, we employ a approach to mixed-species normal mode engineering.

In an array of microtraps individual control over the electric potential at each trap site allows us to engineer normal mode spectra suitable for implementing the desired operators. Previously the use of a special arrangement of the transverse frequencies of individual traps along a string has been proposed for encoding two-body bosonic gauge fields [68]. Here we consider similar ideas to construct phonon modes localized to triplets of neighboring ions (that is, any ion together with both of its nearest neighbors), and with the use of mixed species of ions effectively encode two-body as well as onebody operators. The use of two species of ions allows us to achieve this using mode engineering along only one axis of vibration instead of two transverse axes.

The calculation of the normal modes for a system of ions in an array of microtraps [70,81] involves first determining the equilibrium positions in the combined electric potential due to the trap electrodes and the Coulomb repulsion from the other ions. A Taylor series expansion about these positions up to second order then yields the effective harmonic potential experienced by each ion. The resulting Hamiltonian is then diagonalized to extract the eigenvalues, which give the frequencies of oscillation, and the eigenvectors, which give the relative amplitude of oscillation of each ion in any given normal mode. Since the trapping mechanism in Paul traps and 
(a)

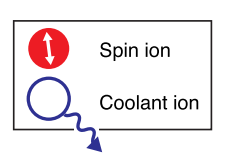

Unperturbed potential wells

Trap site, $k$

Localized modes

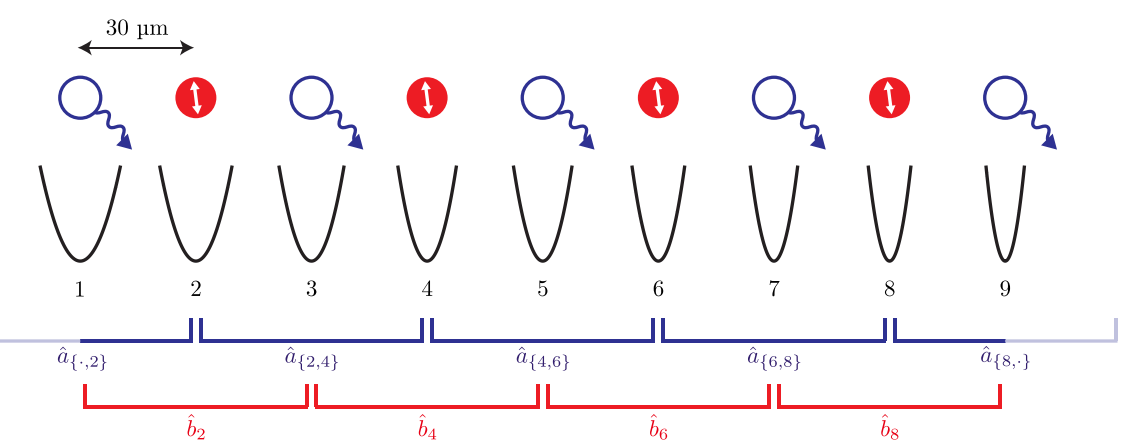

(b)

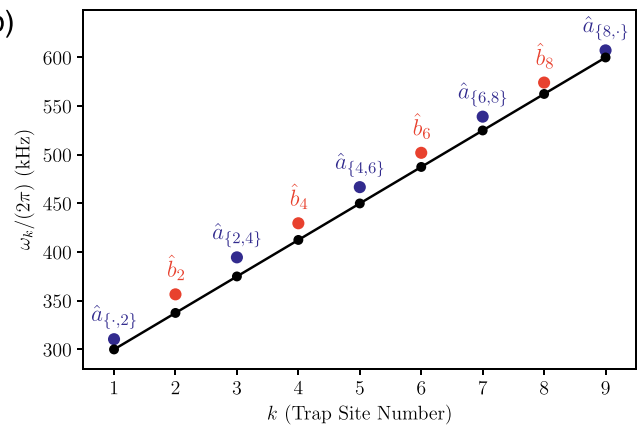

(c)

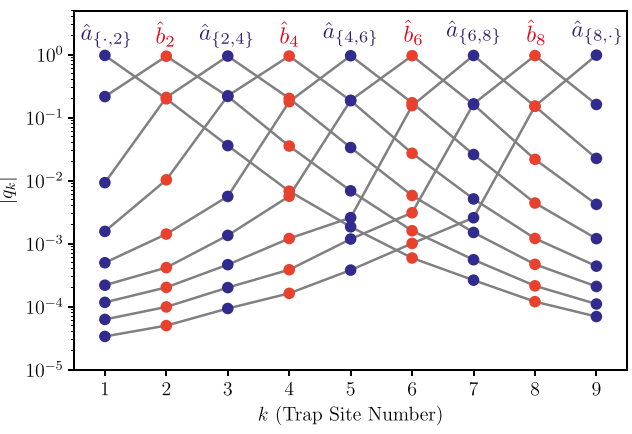

FIG. 12. Mixed-species implementation using microtraps. (a). Ions in a linear chain of microtraps are arranged along the axial direction (this corresponds to the axis with no radio frequency curvature in the case of Paul traps and, equivalently, the magnetic field axis in the case of Penning traps). The distance between neighboring traps here is $30 \mu \mathrm{m}$. Two kinds of ions are considered—one to encode the spins (the spin species, here ${ }^{43} \mathrm{Ca}^{+}$), and the other to facilitate cooling (the coolant species, here ${ }^{40} \mathrm{Ca}^{+}$). Each trap consists of a single ion with the species of the ion occupying the trap sites alternating between the spin and the coolant. By appropriately tuning the individual potential wells one can obtain motional modes localized to either one or two spin ions. (b) Frequency spectrum of the axial modes for an example configuration that leads to the localization of modes. In black are the axial frequencies of the individual microtraps. These vary linearly with the trap site. The colored dots indicate the frequencies of the modes of collective motion for this system of ions. The motion in each mode is at a frequency close to the bare frequency of the trap and is dominated by the ion located at the trap (and its next-nearest-neighbor traps). (c) Eigenvectors of the axial modes showing the amplitudes of motion of each ion in the mode for this configuration. The participation of next-nearest-neighbor (and further) ions drops off steeply in any given mode. Thus, as an approximation, each mode can be treated as being limited to triplets (or pairs in the case of ions at the edge) of ions.

Penning traps differs only in the radial directions, we focus here on the axial modes since the treatment would then be independent of the kind of trap for the discussion below.

To illustrate the idea we first consider a chain of a single species of trapped ions, each with mass $m$. The spatial separation between neighboring traps is given by $d$ and the potentials are designed so that the trap frequencies $\omega_{k}=$ $\omega_{0}+(k-1) \Delta_{\mathrm{T}}$ increase linearly with the trap site $k$. If the frequency difference $\Delta_{\mathrm{T}}$ is much larger than the two-ion exchange frequency, defined by $\Omega_{\mathrm{ex}}=e^{2} /\left(4 \pi \epsilon_{0} m \omega_{0} d^{3}\right)$, we get for this system of coupled ions a spectrum of normal modes close to that of the noninteracting system. That is, the result is a set of modes with oscillation frequency near $\omega_{k}$ and participation mostly from the ion at that trap $k$ (as well as ions at the nearest-neighbor traps, $k-1$ and $k+1$ ).

Now consider a chain of alternating ionic species of nearly equal mass in these traps. One of these species serves as a coolant ion while the other one is used to encode the spins through two suitable internal states, and is from here on called the "spin ion." The condition for nearly equal mass is useful for efficient sympathetic cooling of the spin species through the coolant species. As discussed above, the resulting normal mode structure consists of modes effectively localized to triplets of ions (except, of course, at the edges) but now there exist two kinds of modes-one where the central ion is the coolant ion with two neighboring spin ions, and the other where the central ion is a spin ion between two coolant ions. Figure 12(a) shows this for a chain of alternating ${ }^{40} \mathrm{Ca}^{+}$and ${ }^{43} \mathrm{Ca}^{+}$ions arranged along the axial direction of the microtrap array. The frequencies for the uncoupled arrangement of traps and for the coupled system are shown in Fig. 12(b). Here $\omega_{0}=2 \pi \times 300 \mathrm{kHz}, \Delta_{\mathrm{T}}=2 \pi \times 37.5 \mathrm{kHz}$, and $d=30 \mu \mathrm{m}$. Since the participation of the ions drops roughly exponentially with the distance from the central trap we can assume almost no participation from other ions of the same species (since these lie two sites distant). This behavior can be seen from Fig. 12(c), where the amplitudes of motion of each ion in the axial modes are plotted. Note that each mode is assigned a color so that it is clear which ions dominate the oscillation in a particular mode, and at what frequency. Modes $a$ which are localized to two spin ions (and one coolant ion) allow us to engineer the two-body operators while modes $b$ which are localized to one spin ion (and two coolant ions) allow for implementing one-body operators. To match the notation of Sec. VI, we associate the even-numbered trap sites with the spin ions by $2 k \mapsto j$ (e.g., $a_{2,4} \mapsto a_{1,2}$ ), thereby excluding 
the odd-numbered trap sites containing the coolant ions. Employing sympathetic cooling of these modes, dissipation can be engineered in the $\sigma_{x}$ eigenbasis, following the recipes in Sec. V.

Note that the laser-ion couplings in Sec. VI, Eqs. (44)-(49), require addressing of pairs of ions coherently with the same detuning $\Delta$. As discussed in Sec. IV C, $\Delta$ needs, however, to be matched to the phonon detuning $\delta$, which differs from ion to ion because of the trap frequency offset. The resulting mismatch can be compensated by local AC Stark shifts on $|e\rangle$ and $|f\rangle$, which can be generated by individually addressed lasers.

\section{Alternative realization in linear ion traps}

In conventional bulk ion traps, the desired dynamics can be implemented in a stepwise manner. Here we consider a sequential realization based on delocalized motional modes in combination with local addressing techniques [59,82-85]. We assume all ions to be detuned by a constant amount with respect to the coupling configurations in Sec. VI. For $N$ ions, we now consider $N$ time steps. During each step, we direct a pair of individual addressing lasers on a pair of ions. This beam shifts the transitions of the ions into resonance with the carrier and sideband couplings in Eqs. (44)-(49). We thereby pairwise realize the desired dynamics on ions $j$ and $j+1$, leaving the other ions uncoupled. In the next step, the individual addressing laser is shone onto another pair of ions, $j+1$ and $j+2$, and so forth. Provided short modulation times for the lasers, the timeframes for the ions may be reduced to stroboscopic length, resulting in a "Trotterized" realization of the desired dynamics.

\section{CONCLUSION AND OUTLOOK}

We have presented a scheme suitable to revive the effects of integrability in a controllably driven and open setup, where the underlying Hamiltonian dynamics is only approximately integrable. The scheme is based on weak couplings to Markovian baths in combination with nearly integrable quantum spin Hamiltonians, ingredients which are readily available in state-of-the-art trapped-ion setups. In addition, we present a technique for the engineering of motional modes in an array of mixed-species microtraps, which support the realization of the desired dynamics.

Our numerical analysis shows that despite different sources of integrability breaking due to long-range interactions in the Hamiltonian and openness itself a steady state is realized that cannot be modeled as a thermal ensemble. Instead, approximate expectation values of local observables can be obtained from a generalized Gibbs ensemble and we identify the experimental signatures which reveal that. We presented results for a rotated XY and transverse-field Ising Hamiltonian; however, the same Lindblad operators would activate a GGE in the interacting XXZ Heisenberg chain.

While our goal has been to engineer Lindblad dissipators which stabilize a nontrivial and highly nonthermal steady state by weak driving, a simpler task would be to consider a nontrivial dynamics towards a trivial (e.g., maximally mixed or empty) steady state. As has been shown in Ref. [48], the dynamics of a (nearly) integrable system weakly coupled to arbitrary Lindblad baths can be approximately described with a time-dependent GGE. An example of this is atom loss in cold-atom setups, the effects of which have been observed [86] and theoretically addressed [74,75] very recently. In our setup, the simplest would be to follow the dynamics of an XY Hamiltonian, Eq. (6), in the presence of single-body decay, Eq. (10), alone.

If one had access to several runs of experiments with sufficiently random coupling strengths and/or forms of Lindblad operators to generate a diverse data set of observations, one could also use autoencoder neural networks to detect that more parameters than just temperature are needed for an approximate description of observables [87]. As proposed in the recent Ref. [88], GGEs could emerge also in the Zeno limit of strong dissipations, potentially realized with bosonic trapped gases, and it would be interesting to classify how generic is this mechanism. Another new avenue is to consider Liouville operators that are integrable in the presence of openness $[89,90]$; however, these are experimentally challenging fine tuned models. It would be interesting to study the effect of additional integrability breaking driving in these situations.

Beyond this paper, our dissipation engineering strategies open the door to experiments that will shed light on novel phenomena in open quantum systems. For example, the dissipators presented here have been used to study many-body localization through the perspective of open systems [91]. Furthermore, our mixed-species mode engineering techniques based on arrays of ion microtraps hold promise to become a powerful tool in quantum simulation. Here, sympathetic cooling is not only useful to reduce the entropy of the system, but also to realize complex dissipators. Generalizing these techniques may allow addressing open questions in nonequilibrium quantum many-body physics.

\section{ACKNOWLEDGMENTS}

We thank Achim Rosch, Petar Jurcevic, Christine Maier, and Christian Roos for valuable discussions. This project has received funding from ETH Zürich, from the European Research Council (ERC) under the European Union's Horizon 2020 research and innovation programme Grant Agreement No. 818195, and from the Swiss National Science Foundation through the National Centre of Competence in Research for Quantum Science and Technology (QSIT) grant 51NF40160591, and through the Grant No. BSCGI0 157834. F.R. acknowledges financial support from the Alexander von Humboldt foundation through a Feodor Lynen fellowship and from the Swiss National Science Foundation (Ambizione Grant No. PZ00P2_186040). F.L. acknowledges the financial support of the German Science Foundation under CRC TR 183 (Project No. A01). Z.L. was financially supported by Gordon and Betty Moore Foundation's EPIC initiative, Grant No. GBMF4545; from the European Research Council synergy UQUAM project; and partially by TR CRC 183 (Project No. A01) and Project No. J1-2463 and P1-0044 of the Slovenian Research Agency. Z.L. also acknowledges the L'Oréal-Unesco national scholarship "For women in science" and the NeTex program of University of Cologne, which 
funded visits of Harvard University where this work was initiated.

F.R. and F.L. contributed equally to this work.

\section{APPENDIX A: THEORY OF WEAKLY DRIVEN NEARLY INTEGRABLE SYSTEMS}

Here we review the theory of weakly driven and open nearly integrable systems. We consider the setup discussed in the main text, where the dynamics of the system is given by a dominant integrable Hamiltonian $H_{0}$, in the presence of perturbations of unitary and Markovian nature, which weakly break the integrability. The corresponding Liouvillian terms are

$$
\begin{aligned}
& \mathcal{L}_{0} \rho=-i\left[H_{0}, \rho\right], \quad \mathcal{L}_{u} \rho=-i\left[H_{1}\left(\epsilon_{1}\right), \rho\right], \\
& \mathcal{L}_{m} \rho=\left(\mathcal{D}^{(1)}(\epsilon)+\mathcal{D}^{(2)}(\epsilon)\right) \rho .
\end{aligned}
$$

The steady-state density matrix is determined by

$$
\dot{\rho}_{\infty}=\left(\mathcal{L}_{0}+\mathcal{L}_{p}\right) \rho_{\infty}=0,
$$

where $\mathcal{L}_{p}=\mathcal{L}_{u}+\mathcal{L}_{m}$. Because perturbations due to the Markovian dissipation $\mathcal{L}_{m}$ and the next-nearest-neighbor interaction $\mathcal{L}_{u}$ are only weak, the exact steady-state density matrix $\rho_{\infty}$ can be split as

$$
\rho_{\infty}=\rho_{\mathrm{BD}}+\delta \rho,
$$

where a $\rho_{\mathrm{BD}} \sim \mathcal{O}(1)$, while $\delta \rho\left(\epsilon, \epsilon_{1}\right)$ is a small correction regulated by the strength of unitary and Markovian perturbations. $\rho_{\mathrm{BD}}$ must fulfill the zeroth-order Liouville stationarity equation,

$$
\mathcal{L} \rho_{\infty} \approx \mathcal{L}_{0} \rho_{\mathrm{BD}}=-i\left[H_{0}, \rho_{\mathrm{BD}}\right]=0,
$$

and therefore must have a block-diagonal form

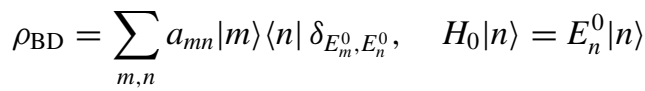

with respect to the eigenstates of $H_{0}$ and is parametrized with about $2^{N}$ parameters $a_{m n}$.

However, it turns out that the description with $\rho_{\mathrm{BD}}$ is redundant and can be replaced with a generalized Gibbs ensemble, $\rho_{\mathrm{BD}} \rightarrow \rho_{\mathrm{GGE}}$, if $H_{0}$ is integrable $[47,48]$ and with a Gibbs ensemble $\rho_{\mathrm{BD}} \rightarrow \rho_{\mathrm{th}}$, if $H_{0}$ is ergodic [92].

While equivalence of $\rho_{\mathrm{BD}}$ and $\rho_{\mathrm{GGE}}$ is formally expected when calculating expectation values of local observables in the thermodynamic limit and with all conservation laws included, in most cases also a tGGE with a few conservation laws

$$
\rho_{\mathrm{tGGE}} \equiv \frac{e^{-\sum_{i=1}^{N_{C}} \lambda_{i} C_{i}}}{\operatorname{tr}\left[e^{-\sum_{i=1}^{N_{C}} \lambda_{i} C_{i}}\right]}
$$

qualitatively well captures the expectation values of local observables, if their support is much smaller than the support of included conservation laws. Known exceptions are observables that are orthogonal to all included conservation laws [93].

In the following we will compare the expectation values evaluated with respect to $\rho_{\infty}, \rho_{\mathrm{BD}}$, and $\rho_{\mathrm{tGGE}}$ in order to confirm that steady states can be approximately described with nonthermal generalized Gibbs ensembles. Note that since our choice of Lindblad operators breaks magnetization, as well as momentum conservation, the whole Hilbert space is of relevance.

The Lagrange parameters $\lambda_{i}$ in Eq. (A5) and parameters $a_{m n}$ in Eq. (A4) are determined from the stationarity conditions in the steady state [47-49]:

$$
\begin{gathered}
\partial_{t} \operatorname{tr}\left[C_{i} \rho_{\mathrm{tGGE}}\right] \stackrel{!}{=} 0, \\
\partial_{t} \operatorname{tr}\left[|m\rangle\langle n| \rho_{\mathrm{BD}}\right] \stackrel{!}{=} 0, \quad E_{m}^{0}=E_{n}^{0}
\end{gathered}
$$

for $\rho_{\mathrm{tGGE}}$ and $\rho_{\mathrm{BD}}$, respectively. For our choice of perturbation the contributions to order $\epsilon$ and $\epsilon_{1}^{2}$

$$
\begin{gathered}
\left\langle\dot{C}_{i}\right\rangle \approx \operatorname{tr}\left[\left(\mathcal{L}_{m}+\mathcal{L}_{u, 2}\right) \rho_{\mathrm{tGGE}}\right] \stackrel{!}{=} 0, \\
\mathcal{L}_{u, 2} \equiv-\mathcal{L}_{u} \mathcal{L}_{0}^{-1} \mathcal{L}_{u}
\end{gathered}
$$

uniquely fix the $\lambda_{i}$ (or equivalently $a_{m n}$ ) in the steady state. Note that $\mathcal{L}_{0} \rho_{\mathrm{tGGE}}=0$ because $\left[H_{0}, C_{i}\right]=0$, and that unitary perturbation contributes to the decay of conservation laws only in the second order, since $\operatorname{tr}\left[C_{i} \mathcal{L}_{u} \rho_{\mathrm{tGGE}}\right]=0$ due to cyclicity of the trace. More details on the derivation of condition (A8) and how to use $\mathcal{L}_{0}^{-1}$ in practice can be found in Ref. [49]. Here we give only the final result for $\left\langle\dot{C}_{i}\right\rangle$ :

$$
\begin{aligned}
\left\langle\dot{C}_{i}\right\rangle= & 2 \pi \sum_{n m}\left(\left\langle m\left|C_{i}\right| m\right\rangle-\left\langle n\left|C_{i}\right| n\right\rangle\right)\left\langle n\left|\rho_{\mathrm{tGGE}}\right| n\right\rangle \\
& \times\left[\left|\left\langle n\left|H_{1}\right| m\right\rangle\right|^{2} \frac{1}{\pi} \frac{\eta}{\left(E_{n}^{0}-E_{m}^{0}\right)^{2}+\eta^{2}}\right. \\
& \left.+\sum_{j}\left(\left|\left\langle n\left|L_{j}^{(1)}\right| m\right\rangle\right|^{2}+\left|\left\langle n\left|L_{j}^{(2)}\right| m\right\rangle\right|^{2}\right)\right]
\end{aligned}
$$

where finite broadening $\eta$ has to be used for calculations at finite system sizes. Expressions relevant for $\rho_{\mathrm{BD}}$ are obtained by replacements $C_{i} \rightarrow\left|m^{\prime}\right\rangle\left\langle n^{\prime}\right| \delta_{E_{m^{\prime}}^{0}, E_{n^{\prime}}^{0}}$ and $\rho_{\mathrm{tGGE}} \rightarrow \rho_{\mathrm{BD}}$.

\section{Numerical results}

We base our analysis on three approaches: (i) calculation of the exact steady state $\rho_{\infty}$, Eq. (A1), at finite but small $\epsilon$, obtained from diagonalization of the full Liouvillian on small system sizes $N=6$ where we exclude or include nextnearest-neighbor (NN) unitary integrability breaking $H_{1}$; (ii) exact calculation of $\rho_{\mathrm{BD}}$, Eq. (A4), on $N=6,8,10$; and (iii) approximate calculation based on a truncated GGE, Eq. (A5), including a finite number of $N_{C}=4$ conservation laws $C_{i}$ on $N=10$. Note that each $C_{i}=\sum_{i} c_{j}^{(i)}$ is a translationally invariant sum of operators $c_{j}^{(i)}$ with support not larger than $i$. Due to finite size effects, only $C_{i}$ with support smaller than $N / 2$ can be included in the tGGE. $C_{i}$ are obtained using the so-called boost operator, $B=-i \sum_{j} j h_{j}$, where $H_{0}=\sum_{j} h_{j}$, from the recursive relation $C_{i+1}=\left[B, C_{i}\right]$ for $i \geqslant 2$ and $C_{2}=$ $H_{0}$. At the isotropic point, $J_{y}=J_{z}$, the magnetization $S^{x}=C_{1}$ is conserved as well.

Figure 13 shows $\left\langle H_{0}\right\rangle$ and $\left\langle C_{4}\right\rangle$ as a function of relative dissipator strength $\gamma$, Eqs. (10) and (11), obtained using different approximations described above at largest accessible system sizes. We observe a good agreement between the three approaches, also for other parameters not displayed. Results 

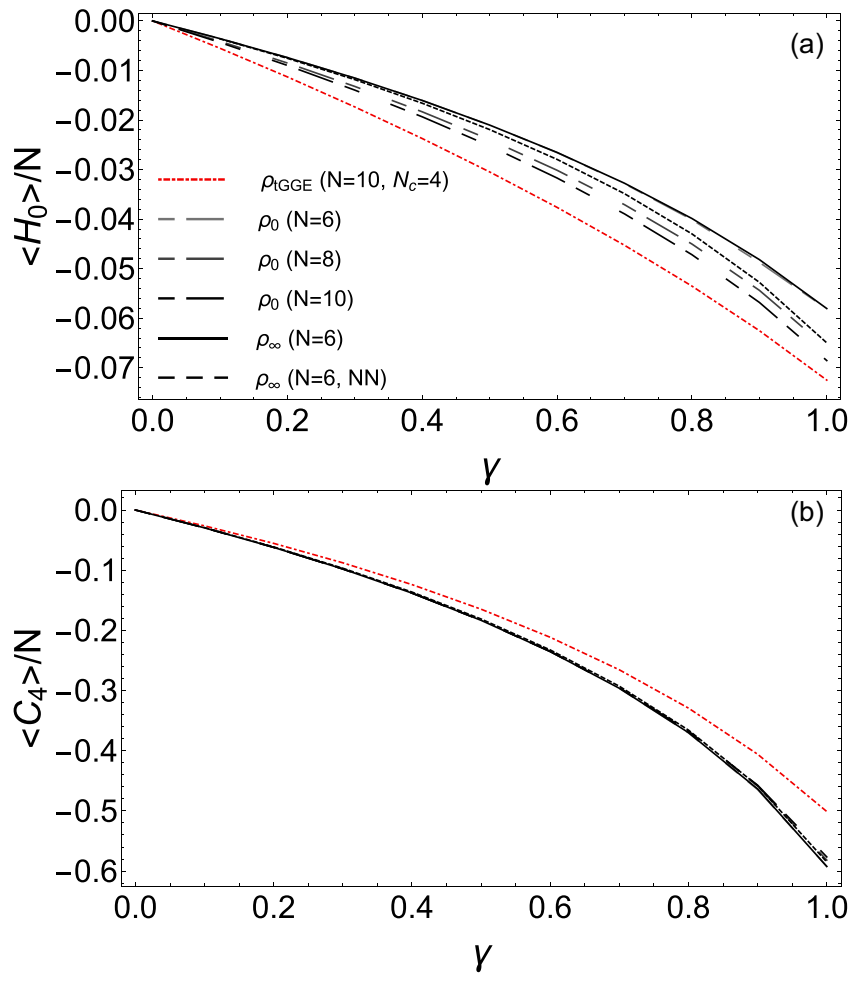

FIG. 13. Expectation values of (a) energy and (b) $C_{4}$ densities as a function of relative driving strength $\gamma$, Eqs. (10) and (11). We compare results calculated (i) with $(\mathrm{NN})$ or without next-nearestneighbor coupling $H_{1}$ from the exact steady state $\rho_{\infty}$ at $\epsilon=0.01$, (ii) from ansatz $\rho_{\mathrm{BD}}$, and (iii) from a truncated GGE $\rho_{\mathrm{tGGE}}$, using $N_{C}=4$ conservation laws. System sizes $N=6,8,10$ are used at $J_{y}=h=1$, $J_{z}=0.1$, and $\epsilon_{1}=0.05$ for $(\mathrm{NN})$.

calculated from $\rho_{\mathrm{BD}}$ on $N=6,8,10$ interpolate between the exact $(N=6)$ and tGGE $(N=10)$ results. While $\rho_{\mathrm{BD}}$ and $\rho_{\infty}$ for small $\epsilon=0.01$ agree very well on $N=6$, increasing the system size shows a tendency of $\rho_{\mathrm{BD}}$ towards the $\rho_{\mathrm{tGGE}}$ result. A milder discrepancy of $\rho_{\mathrm{tGGE}}$ results is due to omitted conservation laws.

The important conclusion is twofold.

(i) The above analysis gives numerical support for the claim that the stabilized steady state can be approximated with a GGE despite different sources of integrability breaking.

(ii) While $\rho_{\mathrm{tGGE}}$ is parametrized with $N_{C}=4$ parameters, $\rho_{\mathrm{BD}}$ at $N=10$ with about $10^{3}$ and the full $\rho_{\infty}$ would require about $10^{6}$ parameters. The description in terms of a truncated GGE is therefore a highly compact parametrization of the steady state, which takes into account only the most relevant information.

We find that in the presence of Lindblad driving the effect of next-nearest-neighbor interaction, $H_{1}$, is rather weak. While in a closed setup $H_{1}$ is crucial as it dictates relaxation towards a thermal state, it is dominated with Lindblad terms in an open setup. Mathematically, this can be explained through Eq. (A10), which shows that the unitary perturbation is constrained to act only between degenerate eigenstates, while the Markovian contribution has no such constraint. Figure 13 shows that results obtained from the exact steady state $\rho_{\infty}$ calculated with $(\mathrm{NN})$ or without $H_{1}$ are very similar. While $H_{1}$ can be easily included into the calculation of the exact steady state $\rho_{\infty}$, it brings certain ambiguity into the calculation of $\rho_{\mathrm{BD}}$ and $\rho_{\mathrm{tGGE}}$. Namely, on finite system sizes one has to introduce broadening $\eta$ when calculating $\mathcal{L}_{u} \mathcal{L}_{0}^{-1} \mathcal{L}_{u}$, Eq. (A10). As we showed in Ref. [48], broadening itself modifies the effective strength of the perturbation, meaning that different system sizes, requiring different broadening, cannot be directly compared. Since $\rho_{\infty}$ shows that the effect of $H_{1}$ is small, we omit it in the calculation of $\rho_{\mathrm{BD}}$ and $\rho_{\mathrm{tGGE}}$.

\section{APPENDIX B: MICROSCOPIC DERIVATION OF TWO-BODY DECAY}

In the following, we verify that the mechanisms presented in Sec. IV C lead to the desired dissipative couplings in Eq. (11). To this end, we eliminate the excited degrees of freedom by means of the effective operator formalism [78]. This allows us to obtain the effective dynamics of the ground states.

To obtain the effective processes between the ground states, we need to evaluate the expressions for the effective Hamiltonian and Lindblad operators [78]:

$$
\begin{gathered}
H_{\mathrm{eff}}=-\frac{1}{2}\left(V_{-} H_{\mathrm{NH}}^{-1} V_{+}+\text {H.c. }\right), \\
L_{\mathrm{eff}, k}=L_{k} H_{\mathrm{NH}}^{-1} V_{+},
\end{gathered}
$$

with the relevant terms discussed below.

For the scheme at hand, $V_{+}$is the weak excitation from the ground states to the excited states (deexcitation: $V_{-}=V_{+}^{\dagger}$ ), taken from Eq. (19):

$$
V_{+}=\frac{\Omega}{2}\left(\sqrt{2}\left|\psi_{e}\right\rangle\langle\downarrow \downarrow|+| \uparrow e\rangle\langle\uparrow \downarrow|\right) .
$$

While $L_{k}$ can represent various sources of dissipation, the only relevant jump operator is the one for induced spontaneous emission Eq. (26). Its essential part acting on $\left|\psi_{e}\right\rangle$ can be written as

$$
L_{\Gamma}=\sqrt{\frac{\Gamma}{2}}|\uparrow \downarrow\rangle\left\langle\psi_{e}\right| .
$$

The evolution of the excited states is described by a nonHermitian Hamiltonian:

$$
H_{\mathrm{NH}}=H_{e}-\frac{i}{2} \sum_{k} L_{k}^{\dagger} L_{k}=H_{\mathrm{e}, \downarrow \downarrow}-\frac{i}{2} L_{\Gamma}^{\dagger} L_{\Gamma},
$$

incorporating the excited-state Hamiltonian $H_{\mathrm{e}, \downarrow \downarrow}$ in Eq. (27) and the jump operator described by Eq. (B4). The nonHermitian terms in Eq. (B5) can then be taken into account by generalizing the detunings from $H_{e}$ to "complex" energies of the form $\tilde{\Delta}=\Delta-i(\Gamma / 2) / 2$. Here we assume no motional decoherence and, hence, $\tilde{\delta}=\delta$. If necessary, processes like phonon decay, $L_{\kappa}=\sqrt{\kappa} a$, can be taken into account by $\tilde{\delta}=$ $\delta-i \kappa / 2$. We obtain

$$
\begin{aligned}
H_{\mathrm{NH}}= & \tilde{\Delta}\left|\psi_{e}\right\rangle|0\rangle\left\langle 0\left|\left\langle\psi_{e}|+\tilde{\delta}| \downarrow \downarrow\right\rangle\right| 1\right\rangle\langle 1|\langle\downarrow \downarrow| \\
& +\sqrt{2} g\left(\left|\psi_{e}\right\rangle|0\rangle\langle 1|\langle\downarrow \downarrow|+| \downarrow \downarrow\rangle| 1\rangle\langle 0|\left\langle\psi_{e}\right|\right) .
\end{aligned}
$$

$H_{\mathrm{NH}}$ is simple to invert:

$$
\begin{aligned}
H_{\mathrm{NH}}^{-1}= & \tilde{\Delta}_{\text {eff }}^{-1}\left|\psi_{e}\right\rangle|0\rangle\left\langle 0\left|\left\langle\psi_{e}\left|+\tilde{\delta}_{\text {eff }}^{-1}\right| \downarrow \downarrow\right\rangle\right| 1\right\rangle\langle 1|\langle\downarrow \downarrow| \\
& +g_{\text {eff }}^{-1}\left(\left|\psi_{e}\right\rangle|0\rangle\langle 1|\langle\downarrow \downarrow|+| \downarrow \downarrow\rangle| 1\rangle\langle 0|\left\langle\psi_{e}\right|\right) .
\end{aligned}
$$


Here we have defined effective detunings and couplings:

$$
\begin{gathered}
\tilde{\Delta}_{\text {eff }}=\tilde{\Delta}-\frac{g^{2}}{\tilde{\delta}}, \\
\tilde{\delta}_{\text {eff }}=\tilde{\delta}-\frac{g^{2}}{\tilde{\Delta}}, \\
\tilde{g}_{\text {eff }}=g-\frac{\tilde{\Delta} \tilde{\delta}}{g},
\end{gathered}
$$

which mediate the effective processes. Using Eq. (B2), we obtain for the effective jump operator for induced spontaneous emission

$$
L_{\mathrm{eff}}^{(2)} \equiv L_{\mathrm{eff}, \Gamma}=L_{\Gamma} H_{\mathrm{NH}}^{-1} V_{+}=\sqrt{\gamma_{2}}|\uparrow \downarrow\rangle|0\rangle\langle 0|\langle\downarrow \downarrow|,
$$

with the effective decay rate

$$
\gamma_{2}=\frac{(\Gamma / 2)(\Omega / \sqrt{2})^{2}}{\left|\tilde{\Delta}_{\text {eff }}\right|^{2}}
$$

For the parameter choice of Sec. IVC $(\Delta=\delta=\sqrt{2} g)$, we find

$$
\tilde{\Delta}_{\text {eff }}=\tilde{\Delta}-\frac{2 g^{2}}{\tilde{\delta}}=-\frac{i \Gamma}{4} .
$$

This yields an effective decay rate:

$$
\gamma_{2} \approx \frac{4 \Omega^{2}}{\Gamma}
$$

We can now associate the effective Lindblad operator in Eq. (B10) with the desired one in Eq. (11),

\section{APPENDIX C: AC STARK SHIFT OF STATES}

We also derive the effective Hamiltonian acting on $|\downarrow \downarrow\rangle|0\rangle$ using Eq. (B1). Here we obtain

$$
H_{\text {eff }}=-\frac{(\Omega / \sqrt{2})^{2}}{\left|\tilde{\Delta}_{\text {eff }}\right|^{2}} \operatorname{Re}\left(\tilde{\Delta}_{\text {eff }}\right)|\downarrow \downarrow\rangle|0\rangle\langle 0|\langle\downarrow \downarrow|,
$$

where $\operatorname{Re}()$ denotes the real part. For our parameter choice $\Delta=\delta=\sqrt{2} g$, we have however that $\operatorname{Re}\left(\tilde{\Delta}_{\text {eff }}\right)=0$. AC Stark shifts can thus be safely neglected in the considered parameter regime.
[1] A. Polkovnikov, K. Sengupta, A. Silva, and M. Vengalattore, Colloquium: Nonequilibrium dynamics of closed interacting quantum systems, Rev. Mod. Phys. 83, 863 (2011).

[2] C. Gogolin and J. Eisert, Equilibration, thermalisation, and the emergence of statistical mechanics in closed quantum systems, Rep. Prog. Phys. 79, 056001 (2016).

[3] M. Rigol, V. Dunjko, V. Yurovsky, and M. Olshanii, Relaxation in a Completely Integrable Many-Body Quantum System: An $A b$ Initio Study of the Dynamics of the Highly Excited States of 1d Lattice Hard-Core Bosons, Phys. Rev. Lett. 98, 050405 (2007).

[4] F. H. L. Essler and M. Fagotti, Quench dynamics and relaxation in isolated integrable quantum spin chains, J. Stat. Mech.: Theory Exp. (2016) P064002.

[5] L. Vidmar and M. Rigol, Generalized Gibbs ensemble in integrable lattice models, J. Stat. Mech.: Theory Exp. (2016) P064007.

[6] M. A. Cazalilla and Ming-Chiang Chung, Quantum quenches in the Luttinger model and its close relatives, J. Stat. Mech.: Theory Exp. (2016) 064004.

[7] J.-S. Caux, The quench action, J. Stat. Mech.: Theory Exp. (2016) P064006.

[8] P. Calabrese and J. Cardy, Quantum quenches in extended systems, J. Stat. Mech.: Theory Exp. (2007) P06008.

[9] M. Cramer, C. M. Dawson, J. Eisert, and T. J. Osborne, Exact Relaxation in a Class of Nonequilibrium Quantum Lattice Systems, Phys. Rev. Lett. 100, 030602 (2008).

[10] T. Barthel and U. Schollwöck, Dephasing and the Steady State in Quantum Many-Particle Systems, Phys. Rev. Lett. 100, 100601 (2008).

[11] F. H. L. Essler, S. Evangelisti, and M. Fagotti, Dynamical Correlations After a Quantum Quench, Phys. Rev. Lett. 109, 247206 (2012).
[12] B. Pozsgay, The generalized Gibbs ensemble for Heisenberg spin chains, J. Stat. Mech.: Theory Exp. (2013) P07003.

[13] M. Fagotti and F. H. L. Essler, Reduced density matrix after a quantum quench, Phys. Rev. B 87, 245107 (2013).

[14] M. Fagotti and F. H. L. Essler, Stationary behaviour of observables after a quantum quench in the spin-1/2 Heisenberg XXZ chain, J. Stat. Mech.: Theory Exp. (2013) P07012.

[15] B. Wouters, J. De Nardis, M. Brockmann, D. Fioretto, M. Rigol, and J.-S. Caux, Quenching the Anisotropic Heisenberg Chain: Exact Solution and Generalized Gibbs Ensemble Predictions, Phys. Rev. Lett. 113, 117202 (2014).

[16] B. Pozsgay, Quantum quenches and generalized Gibbs ensemble in a Bethe Ansatz solvable lattice model of interacting bosons, J. Stat. Mech.: Theory Exp. (2014) P10045.

[17] S. Sotiriadis and P. Calabrese, Validity of the GGE for quantum quenches from interacting to noninteracting models, J. Stat. Mech.: Theory Exp. (2014) P07024.

[18] G. Goldstein and N. Andrei, Failure of the local generalized Gibbs ensemble for integrable models with bound states, Phys. Rev. A 90, 043625 (2014).

[19] M. Brockmann, B. Wouters, D. Fioretto, J. De Nardis, R. Vlijm, and J.-S. Caux, Quench action approach for releasing the néel state into the spin-1/2 XXZ chain, J. Stat. Mech.: Theory Exp. (2014) P12009.

[20] M. Rigol, Quantum quenches in the thermodynamic limit. ii. initial ground states, Phys. Rev. E 90, 031301(R) (2014).

[21] M. Mestyán, B. Pozsgay, G. Takács, and M. A. Werner, Quenching the XXZ spin chain: Quench action approach versus generalized Gibbs ensemble, J. Stat. Mech.: Theory Exp. (2015) P04001.

[22] E. Ilievski, J. De Nardis, B. Wouters, J.-S. Caux, F. H. L. Essler, and T. Prosen, Complete Generalized Gibbs Ensembles in An Interacting Theory, Phys. Rev. Lett. 115, 157201 (2015). 
[23] J. Eisert, M. Friesdorf, and C. Gogolin, Quantum many-body systems out of equilibrium, Nat. Phys. 11, 124 (2015).

[24] E. Ilievski, E. Quinn, J. De Nardis, and M. Brockmann, Stringcharge duality in integrable lattice models, J. Stat. Mech.: Theory Exp. (2016) 063101.

[25] L. Piroli, E. Vernier, and P. Calabrese, Exact steady states for quantum quenches in integrable Heisenberg spin chains, Phys. Rev. B 94, 054313 (2016).

[26] T. Langen, S. Erne, R. Geiger, B. Rauer, T. Schweigler, M. Kuhnert, W. Rohringer, I. E. Mazets, T. Gasenzer, and J. Schmiedmayer, Experimental observation of a generalized Gibbs ensemble, Science 348, 207 (2015).

[27] B. Bertini, F. H. L. Essler, S. Groha, and N. J. Robinson, Prethermalization and Thermalization in Models with Weak Integrability Breaking, Phys. Rev. Lett. 115, 180601 (2015).

[28] B. Bertini, F. H. L. Essler, S. Groha, and N. J. Robinson, Thermalization and light cones in a model with weak integrability breaking, Phys. Rev. B 94, 245117 (2016).

[29] K. Mallayya and M. Rigol, Quantum Quenches and Relaxation Dynamics in the Thermodynamic Limit, Phys. Rev. Lett. 120, 070603 (2018).

[30] K. Mallayya, M. Rigol, and W. De Roeck, Prethermalization and Thermalization in Isolated Quantum Systems, Phys. Rev. X 9, 021027 (2019).

[31] J. Durnin, M. J. Bhaseen, and B. Doyon, Non-equilibrium dynamics and weakly broken integrability, arXiv:2004.11030 (2020).

[32] Y. Tang, W. Kao, K.-Y. Li, S. Seo, K. Mallayya, M. Rigol, S. Gopalakrishnan, and B. L. Lev, Thermalization Near Integrability in A Dipolar Quantum Newton's Cradle, Phys. Rev. X 8, 021030 (2018).

[33] C. Li, T. Zhou, I. Mazets, H.-P. Stimming, F. S. Møller, Z. Zhu, Y. Zhai, W. Xiong, X. Zhou, X. Chen, and J. Schmiedmayer, Relaxation of bosons in one dimension and the onset of dimensional crossover, SciPost Phys. 9, 58 (2020).

[34] J.-S. Caux, B. Doyon, J. Dubail, R. Konik, and T. Yoshimura, Hydrodynamics of the interacting Bose gas in the Quantum Newton Cradle setup, SciPost Phys. 6, 70 (2019).

[35] M. Schemmer, I. Bouchoule, B. Doyon, and J. Dubail, Generalized Hydrodynamics on An Atom Chip, Phys. Rev. Lett. 122, 090601 (2019).

[36] F. Møller, C. Li, I. Mazets, H.-P. Stimming, T. Zhou, Z. Zhu, $X$. Chen, and J. Schmiedmayer, Extension of the Generalized Hydrodynamics to the Dimensional Crossover Regime, Phys. Rev. Lett. 126, 090602 (2021).

[37] M. Moeckel and S. Kehrein, Interaction Quench in the Hubbard Model, Phys. Rev. Lett. 100, 175702 (2008).

[38] M. Kollar, F. A. Wolf, and M. Eckstein, Generalized Gibbs ensemble prediction of prethermalization plateaus and their relation to nonthermal steady states in integrable systems, Phys. Rev. B 84, 054304 (2011).

[39] R. Barnett, A. Polkovnikov, and M. Vengalattore, Prethermalization in quenched spinor condensates, Phys. Rev. A 84, 023606 (2011).

[40] M. Gring, M. Kuhnert, T. Langen, T. Kitagawa, B. Rauer, M. Schreitl, I. Mazets, D. A Smith, E. Demler, and J. Schmiedmayer, Relaxation and prethermalization in an isolated quantum system, Science 337, 1318 (2012).
[41] A. Mitra, Correlation functions in the prethermalized regime after a quantum quench of a spin chain, Phys. Rev. B 87, 205109 (2013).

[42] M. Marcuzzi, J. Marino, A. Gambassi, and A. Silva, Prethermalization in A Nonintegrable Quantum Spin Chain After a Quench, Phys. Rev. Lett. 111, 197203 (2013).

[43] F. H. L. Essler, S. Kehrein, S. R. Manmana, and N. J. Robinson, Quench dynamics in a model with tuneable integrability breaking, Phys. Rev. B 89, 165104 (2014).

[44] N. Nessi, A. Iucci, and M. A. Cazalilla, Quantum Quench and Prethermalization Dynamics in a Two-Dimensional Fermi Gas with Long-Range Interactions, Phys. Rev. Lett. 113, 210402 (2014).

[45] M. Babadi, E. Demler, and M. Knap, Far-from-Equilibrium Field Theory of Many-Body Quantum Spin Systems: Prethermalization and Relaxation of Spin Spiral States in Three Dimensions, Phys. Rev. X 5, 041005 (2015).

[46] E. Canovi, M. Kollar, and M. Eckstein, Stroboscopic prethermalization in weakly interacting periodically driven systems, Phys. Rev. E 93, 012130 (2016).

[47] F. Lange, Z. Lenarčič, and A. Rosch, Pumping approximately integrable systems, Nat. Commun. 8, 15767 (2017).

[48] F. Lange, Z. Lenarčič, and A. Rosch, Time-dependent generalized Gibbs ensembles in open quantum systems, Phys. Rev. B 97, 165138 (2018)

[49] Z. Lenarčič, F. Lange, and A. Rosch, Perturbative approach to weakly driven many-particle systems in the presence of approximate conservation laws, Phys. Rev. B 97, 024302 (2018).

[50] J. T. Barreiro, M. Müller, P. Schindler, D. Nigg, T. Monz, M. Chwalla, M. Hennrich, C. F. Roos, P. Zoller, and R. Blatt, An open-system quantum simulator with trapped ions, Nature (London) 470, 486 (2011).

[51] Y. Lin, J. P. Gaebler, F. Reiter, T. R. Tan, R. Bowler, A. S. Sørensen, D. Leibfried, and D. J. Wineland, Dissipative production of a maximally entangled steady state of two quantum bits, Nature (London) 504, 415 (2013).

[52] D. Leibfried, R. Blatt, C. Monroe, and D. Wineland, Quantum dynamics of single trapped ions, Rev. Mod. Phys. 75, 281 (2003).

[53] D. Porras and J. I. Cirac, Effective Quantum Spin Systems with Trapped Ions, Phys. Rev. Lett. 92, 207901 (2004).

[54] X.-L. Deng, D. Porras, and J. I. Cirac, Effective spin quantum phases in systems of trapped ions, Phys. Rev. A 72, 063407 (2005).

[55] A. Friedenauer, H. Schmitz, J. T. Glueckert, D. Porras, and T. Schaetz, Simulating a quantum magnet with trapped ions, Nat. Phys. 4, 757 (2008).

[56] K. Kim, M.-S. Chang, S. Korenblit, R. Islam, E. E. Edwards, J. K. Freericks, G.-D. Lin, L.-M. Duan, and C. Monroe, Quantum simulation of frustrated Ising spins with trapped ions, Nature (London) 465, 590 (2010).

[57] J. W. Britton, B. C. Sawyer, A. C. Keith, C.-C. J. Wang, J. K. Freericks, H. Uys, M. J. Biercuk, and J. J. Bollinger, Engineered two-dimensional Ising interactions in a trapped-ion quantum simulator with hundreds of spins. Nature (London) 484, 489 (2012).

[58] R. Islam, C. Senko, W. C. Campbell, S. Korenblit, J. Smith, A. Lee, E. E. Edwards, C.-C. J. Wang, J. K. Freericks, and C. Monroe, Emergence and frustration of magnetism with 
variable-range interactions in a quantum simulator, Science 340, 583 (2013).

[59] P. Jurcevic, B. P. Lanyon, P. Hauke, C. Hempel, P. Zoller, R. Blatt, and C. F. Roos, Quasiparticle engineering and entanglement propagation in a quantum many-body system, Nature (London) 511, 202 (2014).

[60] P. Richerme, Z.-X. Gong, A. Lee, C. Senko, J. Smith, M. Foss-Feig, S. Michalakis, A. V. Gorshkov, and C. Monroe, Non-local propagation of correlations in quantum systems with long-range interactions, Nature (London) 511, 198 (2014).

[61] J. G. Bohnet, B. C. Sawyer, J. W. Britton, M. L. Wall, A. M. Rey, M. Foss-Feig, and J. J. Bollinger, Quantum spin dynamics and entanglement generation with hundreds of trapped ions, Science 352, 1297 (2016).

[62] J. Zhang, G. Pagano, P. W. Hess, A. Kyprianidis, P. Becker, H. Kaplan, A. V Gorshkov, Z.-X. Gong, and C. Monroe, Observation of a many-body dynamical phase transition with a 53-qubit quantum simulator, Nature (London) 551, 601 (2017).

[63] C. Kokail, C. Maier, R. van Bijnen, T. Brydges, M. K. Joshi, P. Jurcevic, C. A. Muschik, P. Silvi, R. Blatt, C. F. Roos, and P. Zoller, Self-verifying variational quantum simulation of lattice models, Nature (London) 569, 355 (2019).

[64] A. Safavi-Naini, R. J. Lewis-Swan, J. G. Bohnet, M. Gärttner, K. A. Gilmore, J. E. Jordan, J. Cohn, J. K. Freericks, A. M. Rey, and J. J. Bollinger, Verification of a Many-ion Simulator of the Dicke Model Through Slow Quenches Across a Phase Transition, Phys. Rev. Lett. 121, 040503 (2018).

[65] E. Jordan, K. A. Gilmore, A. Shankar, A. Safavi-Naini, J. G. Bohnet, M. J. Holland, and J. J. Bollinger, Near Ground-State Cooling of Two-Dimensional Trapped-ion Crystals with More than 100 ions, Phys. Rev. Lett. 122, 053603 (2019).

[66] J. I. Cirac and P. Zoller, A scalable quantum computer with ions in an array of microtraps, Nature (London) 404, 579 (2000).

[67] A. C. Wilson, Y. Colombe, K. R. Brown, E. Knill, D. Leibfried, and D. J. Wineland, Tunable spin-spin interactions and entanglement of ions in separate potential wells, Nature (London) 512, 57 (2014).

[68] D. Yang, G. S. Giri, M. Johanning, C. Wunderlich, P. Zoller, and P. Hauke, Analog quantum simulation of $(1+1)$-dimensional lattice QED with trapped ions, Phys. Rev. A 94, 052321 (2016).

[69] M. Mielenz, H. Kalis, M. Wittemer, F. Hakelberg, U. Warring, R. Schmied, M. Blain, P. Maunz, D. L. Moehring, D. Leibfried, and T. Schaetz, Arrays of individually controlled ions suitable for two-dimensional quantum simulations, Nat. Commun. 7, 11839 (2016).

[70] S. Jain, J. Alonso, M. Grau, and J. P. Home, Scalable Arrays of Micro-Penning Traps for Quantum Computing and Simulation, Phys. Rev. X 10, 031027 (2020).

[71] M. D. Barrett, B. DeMarco, T. Schaetz, V. Meyer, D. Leibfried, J. Britton, J. Chiaverini, W. M. Itano, B. Jelenković, J. D. Jost, C. Langer, T. Rosenband, and D. J. Wineland, Sympathetic cooling of ${ }^{9} \mathrm{Be}^{+}$and ${ }^{24} \mathrm{Mg}^{+}$for quantum logic, Phys. Rev. A 68, 042302 (2003).

[72] M. Guggemos, D. Heinrich, O. A. Herrera-Sancho, R. Blatt, and C. F. Roos, Sympathetic cooling and detection of a hot trapped ion by a cold one, New J. Phys. 17, 103001 (2015).
[73] V. Negnevitsky, M. Marinelli, K. K. Mehta, H.-Y. Lo, C. Flühmann, and J. P. Home, Repeated multi-qubit readout and feedback with a mixed-species trapped-ion register, Nature (London) 563, 527 (2018).

[74] I. Bouchoule, B. Doyon, and J. Dubail, The effect of atom losses on the distribution of rapidities in the one-dimensional Bose gas, SciPost Phys. 9, 044 (2020).

[75] A. Hutsalyuk and B. Pozsgay, Integrability breaking in the one dimensional Bose gas: Atomic losses and energy loss, Phys. Rev. E 103, 042121 (2021).

[76] A. Bastianello, J. De Nardis, and A. De Luca, Generalized hydrodynamics with dephasing noise, Phys. Rev. B 102 161110(R) (2020).

[77] K. Yamamoto, Y. Ashida, and N. Kawakami, Rectification in nonequilibrium steady states of open many-body systems, Phys. Rev. Research 2, 043343 (2020).

[78] F. Reiter and A. S. Sørensen, Effective operator formalism for open quantum systems, Phys. Rev. A 85, 032111 (2012).

[79] F. Reiter, D. Reeb, and A. S. Sørensen, Scalable Dissipative Preparation of Many-Body Entanglement, Phys. Rev. Lett. 117, 040501 (2016).

[80] F. Reiter, A. S. Sørensen, P. Zoller, and C. A. Muschik, Dissipative quantum error correction and application to quantum sensing with trapped ions, Nat. Commun. 8, 1822 (2017).

[81] D. V. F. James, Quantum dynamics of cold trapped ions with application to quantum computation, Appl. Phys. B 66, 181 (1998).

[82] P. Schindler, D. Nigg, T. Monz, J. T. Barreiro, E. Martinez, S. X Wang, S. Quint, M. F. Brandl, V. Nebendahl, C. F Roos, M. Chwalla, M. Hennrich, and R. Blatt, A quantum information processor with trapped ions, New J. Phys. 15, 123012 (2013).

[83] U. Warring, C. Ospelkaus, Y. Colombe, R. Jördens, D. Leibfried, and D. J. Wineland, Individual-ion Addressing with Microwave Field Gradients, Phys. Rev. Lett. 110, 173002 (2013).

[84] S. Debnath, N. M. Linke, C. Figgatt, K. A. Landsman, K. Wright, and C. Monroe, Demonstration of a small programmable quantum computer with atomic qubits, Nature (London) 536, 63 (2016).

[85] K. A. Landsman, Y. Wu, P. H. Leung, D. Zhu, N. M. Linke, K. R. Brown, L. Duan, and C. Monroe, Two-qubit entangling gates within arbitrarily long chains of trapped ions, Phys. Rev. A 100, 022332 (2019)

[86] A. Johnson, S. S. Szigeti, M. Schemmer, and I. Bouchoule, Long-lived nonthermal states realized by atom losses in one-dimensional quasicondensates, Phys. Rev. A 96, 013623 (2017).

[87] M. Schmitt and Z. Lenarčič, From observations to complexity of quantum states via unsupervised learning, arXiv:2102.11328 (2021).

[88] D. Rossini, Alexis Ghermaoui, M. B. Aguilera, R. Vatré, R. Bouganne, J. Beugnon, F. Gerbier, and L. Mazza, Strong correlations in lossy one-dimensional quantum gases: From the quantum zeno effect to the generalized Gibbs ensemble, Phys. Rev. A 103, L060201 (2021).

[89] M. de Leeuw, C. Paletta, and B Pozsgay, Constructing Integrable Lindblad Superoperators, Phys. Rev. Lett. 126, 240403 (2021). 
[90] A. Ziolkowska and F. H. L. Essler, Yang-Baxter integrable Lindblad equations, SciPost Phys. 8, 044 (2020).

[91] Z. Lenarčič, O. Alberton, A. Rosch, and E. Altman, Critical Behavior Near the Many-Body Localization Transition in Driven Open Systems, Phys. Rev. Lett. 125, 116601 (2020).
[92] T. Shirai and T. Mori, Thermalization in open many-body systems based on eigenstate thermalization hypothesis, Phys. Rev. E 101, 042116 (2020).

[93] T. Prosen, Open $X X Z$ Spin Chain: Nonequilibrium Steady State and a Strict Bound on Ballistic Transport, Phys. Rev. Lett. 106, 217206 (2011). 